\title{
AN ASYMPTOTIC MODEL FOR COMPRESSION MOLDING
}

\author{
G. Aronsson ${ }^{1}$ \\ DePARTMENT OF MATHEMATiCS \\ LINKÖPING UNIVERSITY \\ LINKÖPING, SWEDEN
}

\author{
L. C. Evans ${ }^{2}$ \\ Department of Mathematics \\ University of CALIFornia, BeRKEley \\ BERKELEY, CA USA
}

\begin{abstract}
We discuss an idealized model for compression molding, had by taking an asymptotic limit for highly non-Newtonian materials. We interpret the changing pressure distributions as being dictated by a Monge-Kantorovich mass transfer on a fast time scale, and thereby derive a nonlocal geometric law of motion for the air/plastic interface.
\end{abstract}

\section{Introduction.}

The deformation of polymer plastic during compression molding is physically complicated, entailing often complex and changing flow patterns, and moving free boundaries. It is consequently interesting to model certain aspects of this behavior by means of simplified mathematical equations. We study in this paper such a model, proposed by the first author (Aronsson [A]) as an asymptotic limiting case of a Hele-Shaw type flow for highly non-Newtonian fluids. The overall point is that the resulting equations, although of course only caricatures of the true physics, nevertheless admit a rigorous and fairly detailed mathematical analysis, displaying velocity and pressure distributions which seem at least qualitatively correct.

Outline. Sections 2 and 3 below recount the derivation of our model from $[\mathrm{A}]$ and $[\mathrm{B}]$, and in particular make clear the many simplifying physical hypotheses. The main idea is to study the material behavior in an asymptotic limit of certain power-law behavior. We as well "hide" explicit mention of the air/plastic free boundary $\Gamma_{t}$ by introducing various multivalued operators. These in fact make possible the subsequent rigorous mathematical analysis.

In $\S 4,5$ we construct a weak or generalized solution to our transformed compression molding problem, starting from an essentially arbitrary initial shape $U_{0}$. This general

\footnotetext{
${ }^{1}$ Supported in part by a Swedish TFR grant, \#97-154.

${ }^{2}$ Supported in part by NSF Grants DMS-9424342 and DMF-0070480.
} 
evolution allows for the merging of different parts of the flow, the disappearance of holes, etc. To build this, we firstly find a sequence of approximations, had by discretizing in time, and along the way carefully record properties of these approximations. In $\S 5$ we send the time step to zero and extract a subsequence of the approximations converging to a weak solution of the full problem. The point is that the corresponding evolution, although highly nonlinear and highly nonlocal, nevertheless gives rise to contractions in the $L^{1}$-norm, provided we turn attention to $w$, the indicator function of the plastic region. Theorem 5.1 records our main existence and uniqueness assertion.

The difficult mathematics of $\S 4,5$ done with, we turn in the remaining sections to working out some further formal, unproved consequences of our theory, and most importantly derive in $§ 6$ a nonlocal geometric law of evolution for the free boundary. This reads:

$$
V=\gamma\left(1-\frac{\kappa \gamma}{2}\right)
$$

for

$$
\left\{\begin{array}{l}
V=\text { outward normal velocity of the free boundary } \Gamma_{t} \\
\gamma=\text { ridge distance } \\
\kappa=\text { curvature of } \Gamma_{t},
\end{array}\right.
$$

the ridge being, roughly speaking, the set of points within the plastic region equidistant to two or more closest boundary points. The term "ridge distance" is explained in $\S 6$. Bergwall [B1, B2] earlier obtained (1.1), (1.2) by a different argument.

In $\S 7$ we specialize our rule $(1.1),(1.2)$ to various special geometric situations, deriving several nontrivial, and perhaps testable, predictions about the free boundary motion. The concluding $\S 8$ provides some interesting geometric calculations for general, smooth flows governed by (1.1), (1.2).

Remarks. Many of the subsequent calculations, both rigorous and formal, are inspired by ideas originating with the Monge-Kantorovich mass transfer problem, as explained in the survey $[\mathrm{E}]$ and the long paper [E-G1]. See also the recent papers Ambrosio [Am] and Caffarelli-Feldman-McCann [C-F-M] for much more.

Some other related papers are Elliott-Janovsky [E-J], Folgar-Lee-Tucker [F-L-T], Janfalk $[\mathrm{J} 1,2]$, King $[\mathrm{K}]$ and Osswald-Tucker $[\mathrm{O}-\mathrm{T}]$

We thank the referee for carefully reading our paper.

\section{Derivation of the model.}

This section provides a recounting of the derivation in Aronsson [A] and Bergwall [B1] of our model problem. There will be many simplifying assumptions. 
a. Geometric notation. We suppose that at each moment of time $t$, the compressed plastic lies between two infinite horizontal plates, the lower at height zero and the upper at height $h=h(t)>0$.

Let us assume

$$
\dot{h}(t)<0 \text { for } 0 \leq t<T \text {, }
$$

$T \leq \infty$ being the time when the two plates meet.

For each $0 \leq t<T$, denote by $U_{t}$ the open subregion above which the plastic lies. That is, we assume that at time $t$ the plastic occupies the volume $U_{t} \times(0, h) \subset \mathbb{R}^{3}$, at least approximately. Then

$$
\Gamma_{t}:=\partial U_{t}
$$

represents the projection of the air/plastic interface onto $\mathbb{R}^{2}$. A basic problem will be to track $\left\{\Gamma_{t}\right\}_{t \geq 0}$ as time evolves.

b. Velocity, pressure, strain and stress. We write $\mathbf{v}=(u, v, w)$ for the material velocity and $p$ for the pressure. The linearized strain rate tensor (or stretching tensor) is

$$
\boldsymbol{\Sigma}:=\frac{D \mathbf{v}+D \mathbf{v}^{T}}{2}=\frac{1}{2}\left(\begin{array}{ccc}
2 u_{x} & u_{y}+v_{x} & u_{z}+w_{x} \\
u_{y}+v_{x} & 2 v_{y} & v_{z}+w_{y} \\
u_{z}+w_{x} & v_{z}+w_{y} & 2 w_{z}
\end{array}\right)
$$

The stress tensor $\mathbf{T}$ has the form

$$
\mathbf{T}=-p \mathbf{I}+\hat{\mathbf{T}}
$$

and we hypothesize that the deviatoric stress $\hat{\mathbf{T}}$ is given by the constitutive law

$$
\hat{\mathbf{T}}=\eta(|\boldsymbol{\Sigma}|) \boldsymbol{\Sigma}
$$

where $|\boldsymbol{\Sigma}|^{2}=\operatorname{tr}\left(\boldsymbol{\Sigma} \boldsymbol{\Sigma}^{T}\right)$. We further assume $\eta$ to have the power-law structure

$$
\eta(|\boldsymbol{\Sigma}|)=K|\boldsymbol{\Sigma}|^{\sigma-1}
$$

$K$ and $\sigma$ denoting positive material constants.

The full flow equations read

$$
\left\{\begin{array}{l}
\rho \frac{D \mathbf{v}}{D t}=\operatorname{div}(\mathbf{T})+\rho \mathbf{g} \\
\operatorname{div} \mathbf{v}=0
\end{array} \quad \text { in } U_{t} \times(0, h),\right.
$$

where $\rho$ denotes the density and $\mathbf{g}=(0,0,-g)$ is the gravity vector. The operator $\frac{D}{D t}:=$ $\frac{\partial}{\partial t}+u \frac{\partial}{\partial x}+v \frac{\partial}{\partial y}+w \frac{\partial}{\partial z}$ is the usual material derivative. 
c. Averages. Since we will assume $0<h \ll 1$, we expect the first two components of the velocity vector $\mathbf{v}=(u, v, w)$ to be physically most important. It therefore seems appropriate to introduce the height averages:

$$
\left\{\begin{array}{l}
\bar{u}(x, y, t):=\frac{1}{h} \int_{0}^{h} u(x, y, z, t) d z \\
\bar{v}(x, y, t):=\frac{1}{h} \int_{0}^{h} v(x, y, z, t) d z
\end{array} \quad(x, y) \in U_{t} .\right.
$$

Physically we should have $w=0$ along the fixed bottom plate $\{z=0\}$, but $w=\dot{h}$ along the moving top plate $\{z=h\}$. Consequently the identity div $\mathbf{v}=0$ transforms to read

$$
\bar{u}_{x}+\bar{v}_{y}=-\frac{\dot{h}}{h} \quad \text { in } U_{t}
$$

d. Hele-Shaw approximations. We next dramatically simplify the flow equations (2.7) by additionally hypothesizing that viscosity effects and pressure gradient effects predominate, in accordance with the usual Hele-Shaw assumptions. In particular, we ignore the acceleration term $\frac{D \mathbf{v}}{D t}$ and the body force $\mathbf{g}$ in (2.7). Then (2.3), (2.4) and (2.7) imply

$$
D p=\operatorname{div}(\eta(|\boldsymbol{\Sigma}|) \boldsymbol{\Sigma}) \quad \text { in } U_{t} \times(0, h),
$$

$D$ denoting the gradient with respect to the spatial variables. We further simplify by assuming the pressure $p$ does not depend on $z$ and that $w$ may henceforth be taken to be zero in computing $\Sigma$. Additionally, as the velocities $u, v$ are 0 on $\{z=0, h\}$, we predict that the derivatives $u_{z}, v_{z}$ are much larger than $u_{x}, u_{y}, v_{x}, v_{y}$, and consequently that the latter may be ignored within the stretching tensor $\boldsymbol{\Sigma}$. Incorporating all these simplifying hypotheses into (2.10) yields the identities

$$
\left\{\begin{array}{l}
p_{x}=\left(\eta u_{z}\right)_{z} \\
p_{y}=\left(\eta v_{z}\right)_{z}
\end{array}\right.
$$

As $p$, and so $p_{x}, p_{y}$, do not depend on $z$, we conclude

$$
\left\{\begin{array}{l}
u_{z}=\frac{p_{x}}{\eta}\left(z-\frac{h}{2}\right) \\
v_{z}=\frac{p_{y}}{\eta}\left(z-\frac{h}{2}\right)
\end{array}\right.
$$

owing to symmetry about the level $\{z=h / 2\}$. Now

$$
\begin{aligned}
\eta & =\eta\left(\left(u_{z}^{2}+v_{z}^{2}\right)^{1 / 2}\right)=K\left(u_{z}^{2}+v_{z}^{2}\right)^{\frac{\sigma-1}{2}} \quad \text { by }(2.6) \\
& =\frac{K}{\eta^{\sigma-1}}\left|z-\frac{h}{2}\right|^{\sigma-1}|D p|^{\sigma-1} \quad \text { by }(2.11) ;
\end{aligned}
$$


whence

$$
\eta=K^{\frac{1}{\sigma}}\left|z-\frac{h}{2}\right|^{1-\frac{1}{\sigma}}|D p|^{1-\frac{1}{\sigma}} .
$$

We insert this equality into (2.11) and integrate, to deduce

$$
\left\{\begin{array}{l}
u=-\Phi|D p|^{\frac{1}{\sigma}-1} p_{x} \\
v=-\Phi|D p|^{\frac{1}{\sigma}-1} p_{y}
\end{array}\right.
$$

for

$$
\Phi:=A\left(h^{\frac{1}{\sigma}+1}-|h-2 z|^{\frac{1}{\sigma}+1}\right) \quad(0 \leq z \leq h)
$$

and $A$ an appropriate material constant. Hence

$$
\left\{\begin{array}{l}
\bar{u}=-B|D p|^{\frac{1}{\sigma}-1} p_{x} \\
\bar{v}=-B|D p|^{\frac{1}{\sigma}-1} p_{y}
\end{array}\right.
$$

for another constant $B$. Recalling then the incompressibility condition (2.9) we conclude

$$
\operatorname{div}\left(|D p|^{\frac{1}{\sigma}-1} D p\right)=\frac{\dot{h}}{h} \quad \text { within } U_{t},
$$

where we have rescaled to set all constants equal to one.

e. Rescaling time. We effect a further simplification by hereafter supposing

$$
\frac{\dot{h}}{h} \equiv-1, \quad T=+\infty .
$$

Indeed if (2.14) fails, we can change variables in time by writing $t=\theta(s)(0 \leq s<\infty), \theta$ solving the ODE

$$
\left\{\begin{array}{l}
\theta^{\prime}(s)=-\frac{h(\theta(s))}{\dot{h}(\theta(s))} \quad(0<s<\infty) \\
\theta(0)=0 .
\end{array}\right.
$$

Then $\theta^{\prime}>0, \lim _{s \rightarrow \infty} \theta(s)=T$, and $(2.14)$ holds provided we reinterpret ${ }^{\cdot}=\frac{d}{d s}, h=$ $h(\theta(s))$. In view of (2.14), our PDE now becomes

$$
-\operatorname{div}\left(|D p|^{\frac{1}{\sigma}-1} D p\right)=1 \quad \text { in } U_{t} .
$$

f. Boundary conditions. Finally on the air/plastic interface $\Gamma_{t}=\partial U_{t}$ let us suppose

$$
p=0 \quad \text { on } \Gamma_{t},
$$

after our having subtracted atmospheric pressure. We also take

$$
V=|D p|_{5}^{\frac{1}{\sigma}} \quad \text { on } \Gamma_{t}
$$


$V$ denoting the outward normal velocity of the free boundary $\Gamma_{t}$. Since the unit outer normal field is $\boldsymbol{\nu}=-\frac{D p}{|D p|}$ on $\Gamma_{t},(2.17)$ simply asserts that the leading edge of the deforming plastic moves with the velocity of the flow.

Remark. Let us pause here to check a consequence of our model thus far. We compute

$$
\begin{aligned}
\frac{d}{d t}\left|U_{t}\right| & =\int_{\Gamma_{t}} V d \mathcal{H}^{1}=-\int_{\Gamma_{t}}|D p|^{\frac{1}{\sigma}} \frac{D p}{|D p|} \cdot \nu d \mathcal{H}^{1} \\
& =-\int_{U_{t}} \operatorname{div}\left(|D p|^{\frac{1}{\sigma}-1} D p\right) d x=\left|U_{t}\right| .
\end{aligned}
$$

In this calculation $\mathcal{H}^{1}$ denotes one-dimensional Hausdorff measure. Therefore in view of (2.14), we derive the conservation of mass assertion:

$$
\frac{d}{d t}\left(h(t)\left|U_{t}\right|\right)=0 .
$$

g. Sending $\boldsymbol{\sigma} \rightarrow \boldsymbol{0}$. Our compression model thus far comprises the PDE (2.15), with the free boundary conditions $(2.16),(2.17)$. This is a " $p$-Laplacian" version of a particular form of the Hele-Shaw flow.

We intend next again to simplify the structure by taking the limit $\sigma \rightarrow 0$, corresponding to an extremely shear-thinning flow, supporting, as we shall see, "infinitely fast/infinitely slow" mass transfer effects. Our rationale here is severalfold. First and foremost, we will see that the resulting asymptotic problem is much simpler in structure than the corresponding flow for $\sigma>0$. Roughly speaking, as $\sigma \rightarrow 0$ in (2.15) the viscous effects become "instantaneous" and consequently easier to monitor. Much of the analysis will therefore drastically simplify, and we will accordingly be able to obtain various ODE and/or geometric motion laws for $\Gamma_{t}$ that exactly describe the limit flows. In addition the resulting mathematics is fairly elegant. We will for instance find that the flows, although highly nonlinear, correspond to contractions in $L^{1}$. Lastly, taking the limit $\sigma \rightarrow 0$ is supported by numerical and experimental evidence. For instance, Folgar, Lee and Tucker in [F-L-T] report that the flow patterns do not much depend on $\sigma$, once $\sigma$ is sufficiently small.

In the next section we scrutinize our problems (2.15)-(2.17) in the limit $\sigma \rightarrow 0$.

\section{Formal asymptotic limits as $\sigma \rightarrow 0$.}

a. Asymptotics. Again following Aronsson [A], we propose now to analyze the limit $\sigma \rightarrow 0$ of our compression problem (2.15)-(2.17). First of all, let us transform notation, so as to be consistent with the mathematics references. Hereafter we introduce the parameter $p$ according to

$$
\frac{1}{\sigma}-1=p-2
$$


and then write $u_{p}=u_{p}\left(x_{1}, x_{2}, t\right)$, for $x=\left(x_{1}, x_{2}\right)$, to denote the pressure. Thus our sending $\sigma \rightarrow 0$ is equivalent to letting $p \rightarrow \infty$.

Fix a time $t \geq 0$, and consider the PDE

$$
\left\{\begin{aligned}
-\operatorname{div}\left(\left|D u_{p}\right|^{p-2} D u_{p}\right)=1 & \text { in } U_{t} \\
u_{p}=0 & \text { on } \Gamma_{t}
\end{aligned}\right.
$$

where for the moment we ignore the dependence of the free boundary $\Gamma_{t}$ (and thus $U_{t}$ ) on $p$ and we temporarily ignore as well the motion in time of $\Gamma_{t}$. It is convenient to recast (3.1) into abstract form, by writing

$$
\partial I_{p}^{t}\left[u_{p}\right]=1
$$

for

$$
I_{p}^{t}[v]:= \begin{cases}\frac{1}{p} \int_{U_{t}}|D v|^{p} d x & \text { if } v \in W_{0}^{1, p}\left(U_{t}\right) \\ +\infty & \text { otherwise. }\end{cases}
$$

The functional $I_{p}^{t}[\cdot]$ is convex and lower semicontinuous on $L^{2}\left(U_{t}\right)$. The precise meaning of $(3.2)$ is that

$$
I_{p}^{t}[v] \geq I_{p}^{t}[u(\cdot, t)]+\int_{U_{t}} v-u(\cdot, t) d x
$$

for all $v \in L^{2}\left(U_{t}\right)$.

The reformulation (3.2), (3.3) suggests that we can compute the limit $p \rightarrow \infty$ of (3.1) by setting

$$
I_{\infty}^{t}[v]:= \begin{cases}0 & \text { if } v \in W_{0}^{1, \infty}\left(U_{t}\right) \text { and }|D v| \leq 1 \text { a.e. } \\ +\infty & \text { otherwise. }\end{cases}
$$

Thus we interpret the problem resulting from (3.1) in the limit $p \rightarrow \infty$ as saying

$$
1 \in \partial I_{\infty}^{t}[u]
$$

provided $u_{p} \rightarrow u$. This means that

$$
I_{\infty}^{t}[v] \geq I_{\infty}^{t}[u(\cdot, t)]+\int_{U_{t}} v-u(\cdot, t) d x
$$

for all $v$ as above. We also expect

$$
|D u| \leq 1 \text { a.e. in } U_{t}
$$

Furthermore, according to the general mass transfer theory (as explained for instance in $[\mathrm{E}])$, there exists for each time $t>0$ a function $a$ such that

$$
-\operatorname{div}(a D u)=1 \quad \text { in } U_{t}
$$


in the weak sense, which means

$$
\int_{U_{t}} a D u \cdot D v d x=\int_{U_{t}} v d x
$$

for each $v \in C_{c}^{1}\left(U_{t}\right)$. This function $a$ may be interpreted as the Lagrange multiplier corresponding to the gradient constraint (3.5). In addition,

$$
a \geq 0 \text { a.e., } \quad \operatorname{spt}(\mathrm{a}) \subset\{|D u|=1\} \text {. }
$$

The physical interpretation of all this is that in the limit $\sigma \rightarrow 0$ :

$$
\left\{\begin{array}{l}
u \text { is the pressure, } \\
\mathbf{v}=-a D u \text { is the material velocity } \\
a=|\mathbf{v}| \text { is the speed. }
\end{array}\right.
$$

Consequently we expect the conditions along the free boundary $\Gamma_{t}$ to read

$$
u=0, a=V \text { on } \Gamma_{t},
$$

$V$ as before denoting the outer normal velocity.

We summarize: the formal limit as $p \rightarrow \infty$ (i.e. $\sigma \rightarrow 0$ ) of our compression molding problem is (3.5)-(3.9), where the pressure $u$, the speed $a$, and the free boundaries $\left\{\Gamma_{t}\right\}_{t \geq 0}$ are the unknowns.

b. The pressure as distance function. This is still a difficult generalized HeleShaw type free boundary problem. However our central thesis is that in fact the structure of problem (3.5)-(3.9) is not really so complicated: various simplifications have actually occurred in the $p \rightarrow \infty$ limit. We illustrate this principle by noting explicitly that

$$
u(x, t)=\operatorname{dist}\left(x, \Gamma_{t}\right) \quad\left(x \in U_{t}\right) .
$$

In other words, in the $p \rightarrow \infty$ asymptotic limit the pressure $u$ is recovered immediately from the shape of the plastic region at each time $t \geq 0: u$ is simply the shortest distance to the air/plastic interface $\Gamma_{t}$. To motivate (3.10) we note that (3.2) says $u_{p}$ minimizes

$$
\int_{U_{t}} \frac{1}{p}|D v|^{p}-v d x
$$

over all $v \in W_{0}^{1, p}\left(U_{t}\right)$. Consequently $u=\lim _{p \rightarrow \infty} u_{p}$ should maximize

$$
\int_{U_{t}} v d x
$$


over all $v \in W_{0}^{1, p}\left(U_{t}\right)$, subject to the constraint $|D v| \leq 1$ a.e. The distance function $u$ defined by (3.10) is the unique solution of this maximization problem.

c. Free boundary motion. Next we modify the foregoing considerations, explicitly taking into account the motion of the free boundary $\Gamma_{t}$. Following, for instance, ElliottOckendon [E-O], we continue to adopt an abstract viewpoint, but now consider in place of (3.1) the full evolution in time:

$$
\left\{\begin{aligned}
-\operatorname{div}(a D u) & =1 \quad \text { in } U_{t} \\
u(x, t) & =\operatorname{dist}\left(x, \Gamma_{t}\right) \quad \text { in } U_{t} \\
V & =a \quad \text { on } \Gamma_{t} .
\end{aligned}\right.
$$

Let us extend $u$ to be zero in $\mathbb{R}^{2}-U_{t}$, and write

$$
w:=\chi_{U_{t}}
$$

to denote the indicator function, equal to 1 on $U_{t}$ and 0 on $\mathbb{R}^{2}-U_{t}$.

We claim next that the evolution (3.11) can be formally rewritten as

$$
w-w_{t} \in \partial I_{\infty}[u]
$$

where now

$$
I_{\infty}[v]:= \begin{cases}0 & \text { if } v \in L^{2}\left(\mathbb{R}^{2}\right),|D v| \leq 1 \text { a.e. } \\ +\infty & \text { otherwise. }\end{cases}
$$

Observe there is no reference to the set $U_{t}$ in the definition of $I_{\infty}[\cdot]$.

To confirm (3.13), let us suppose $u, a,\left\{\Gamma_{t}\right\}_{t \geq 0}$ comprise a sufficiently smooth solution of (3.11) to justify the following computations. Take $\phi$ to be a smooth test function with compact support in $\mathbb{R}^{2} \times(0, \infty)$. Then

$$
0=\int_{0}^{\infty} \frac{d}{d t}\left(\int_{U_{t}} \phi d x\right) d t=\int_{0}^{\infty} \int_{U_{t}} \phi_{t} d x d t+\int_{0}^{\infty} \int_{\Gamma_{t}} \phi V d \mathcal{H}^{1} d t
$$

$\mathcal{H}^{1}$ denoting one-dimensional Hausdorff measure. But the first line of (3.11) implies for each fixed time $t$ that

$$
\int_{U_{t}} \phi d x=-\int_{U_{t}} \phi \operatorname{div}(a D u) d x=\int_{U_{t}} a D u \cdot D \phi d x-\int_{\Gamma_{t}} \phi a \frac{\partial u}{\partial \nu} d \mathcal{H}^{1},
$$

$\nu$ being the unit outward normal. Observe $\frac{\partial u}{\partial \nu} \equiv-1$ on $\Gamma_{t}$, owing to (3.10). Since $V=a$ on $\Gamma_{t}$, we can combine (3.14), (3.15), thereby obtaining the identity:

$$
\begin{aligned}
0 & =\int_{0}^{\infty} \int_{U_{t}} \phi_{t}+\phi-a D u \cdot D \phi d x d t \\
& =\int_{0}^{\infty} \int_{\mathbb{R}^{2}} w \phi_{t}+w \phi-a D u \cdot D \phi d x d t,
\end{aligned}
$$


the last equality holding since both $u$ and $w$ vanish outside $U_{t}$.

As the foregoing equality is valid for each $\phi$ as above, we deduce that

$$
w_{t}-\operatorname{div}(a D u)=w \quad \text { in } \mathbb{R}^{2} \times(0, \infty)
$$

in the weak sense. But if $|D v| \leq 1$ and $v$ has compact support on $\mathbb{R}^{2}$, then formally at least,

$$
\int_{\mathbb{R}^{2}}-\operatorname{div}(a D u)(v-u) d x=\int_{U_{t}} a\left(D u \cdot D v-|D u|^{2}\right) d x \leq 0,
$$

since $a \geq 0$ and $|D u|=1$ a.e. in $U_{t}$. Consequently $w-w_{t}=-\operatorname{div}(a D u) \in \partial I_{\infty}[u]$. Hence our assertion (3.13) follows from (3.16).

d. Weak solutions. The advantage of the formulation (3.13) is that the regions $U_{t}$ and their free boundaries $\Gamma_{t}$ no longer explicitly appear. Furthermore, as demonstrated in $\S 4,5$ following, the evolution (3.13) is well-posed in an appropriately weak sense, provided we regard $w$, and not $u$, as the principal unknown. The mapping $t \mapsto w$ turns out to be fairly well behaved, although the mapping $t \mapsto u$ need not be: see Example $\mathrm{A}$ in $\S 7$.

So, finally to rewrite (3.13) solely in terms of $w$, we introduce the multivalued graph

$$
\beta(r):= \begin{cases}\emptyset & \text { if } r<0 \\ (-\infty, 0] & \text { if } r=0 \\ 0 & \text { if } 0<r<1 \\ {[0, \infty)} & \text { if } r=1 \\ \emptyset & \text { if } r>1\end{cases}
$$

Then for each time $t, u \in \beta(w)$, owing to (3.12).

\section{Approximation.}

To summarize, we have refashioned our compression problem into the multivalued evolution

$$
\left\{\begin{array}{l}
w-w_{t} \in \partial I_{\infty}[u] \\
u \in \beta(w)
\end{array} \quad(t>0),\right.
$$

with

$$
w=\chi_{U_{0}} \quad(t=0)
$$

Hereafter assume $U_{0}$ is a bounded open subset of $\mathbb{R}^{2}$, with boundary $\partial U_{0}$ having twodimensional Lebesgue measure zero: $\left|\partial U_{0}\right|=0$. We propose to build a solution of (4.1), (4.2) as the limit as $h \rightarrow 0^{+}$of the discrete time approximations

$$
\left\{\begin{array}{l}
w^{k+1}+h \partial I_{\infty}\left[u^{k+1}\right] \ni(1+h) w^{k} \\
u^{k+1} \in \beta\left(w^{k+1}\right), u_{10}^{k+1} \geq 0
\end{array} \quad(k=0, \ldots),\right.
$$


where

$$
w^{0}=\chi_{U_{0}}
$$

It will be convenient sometimes to rewrite (4.3) as

$$
\alpha\left(u^{k+1}\right)+h \partial I_{\infty}\left[u^{k+1}\right] \ni(1+h) w^{k}
$$

for the multivalued graph $\alpha:=\beta^{-1}$; that is,

$$
\alpha(r):= \begin{cases}0 & \text { if } r<0 \\ {[0,1]} & \text { if } r=0 \\ 1 & \text { if } r>0\end{cases}
$$

Proposition 4.1. (i) For each $k=0,1, \ldots$, there exists a pair $\left(u^{k+1}, w^{k+1}\right)$ of bounded, measurable functions with compact support, which solve (4.3).

(ii) Furthermore,

$$
u^{k+1} \text { is Lipschitz continuous, } u^{k+1} \geq 0,\left|D u^{k+1}\right|=1 \text { a.e. on } U_{k+1}
$$

and

$$
w^{k+1}=\chi_{U_{k+1}},
$$

for

$$
U_{k+1}:=\left\{u^{k+1}>0\right\}
$$

(iii) We also have

$$
\left|\partial U_{k+1}\right|=0
$$

and

$$
\left|U_{k+1}\right|=(1+h)\left|U_{k}\right|
$$

(iv) If $U_{k} \subseteq B(0, R)$, then

$$
U_{k+1} \subseteq B\left(0,(1+h)^{1 / 2} R\right) .
$$


(v) The pair $\left(u^{k+1}, w^{k+1}\right)$ is unique among functions with the foregoing properties.

Proof. 1. The proof is by induction. Assume therefore for some nonnegative integer $k$ that

$$
w^{k}=\chi_{U_{k}}
$$

where $U_{k}$ is open, bounded, and $\left|\partial U_{k}\right|=0$. We consider the problem (4.3) in the transformed version (4.5), which in turn we recognize as the Euler-Lagrange equation for the variational problem of minimizing the functional

$$
J_{k+1}[v]:=\int_{\mathbb{R}^{2}} v^{+}-(1+h) v w^{k} d x=\int_{\mathbb{R}^{2}} v^{+} d x-(1+h) \int_{U_{k}} v d x
$$

among Lipschitz continuous functions $v$ with $|D v| \leq 1$ a.e.. It is not hard to show that there exists a minimizer $u^{k+1}$. Replacing $u^{k+1}$ by $\left(u^{k+1}\right)^{+}$if needs be, we may suppose

$$
u^{k+1} \geq 0
$$

Define the open set

$$
U_{k+1}:=\left\{u^{k+1}>0\right\}
$$

2. We assert first that

$$
U_{k+1} \supseteq U_{k}
$$

To prove this, note that since $u^{k+1} \geq 0$,

$$
J_{k+1}\left[u^{k+1}\right]=\int_{\mathbb{R}^{2}-U_{k}} u^{k+1} d x-h \int_{U_{k}} u^{k+1} d x .
$$

Define

$$
\bar{u}^{k+1}(x):= \begin{cases}u^{k+1}(x) & \left(x \in \mathbb{R}^{2}-U_{k}\right) \\ \min _{y \in \mathbb{R}^{2}-U_{k}}\left\{u^{k+1}(y)+|x-y|\right\} & \left(x \in U_{k}\right) .\end{cases}
$$

Then $\left|D \bar{u}^{k+1}\right| \leq 1$ a.e., $\bar{u}^{k+1} \equiv u^{k+1}$ on $\mathbb{R}^{2}-U_{k}$, and $\bar{u}^{k+1} \geq u^{k+1}$ on $U_{k}$. Were $\bar{u}^{k+1}(x)>$ $u^{k+1}(x)$ for some point $x \in U_{k}$, we would derive from (4.17) the contradiction

$$
J_{k+1}\left[\bar{u}^{k+1}\right]<J_{k+1}\left[u^{k+1}\right]
$$

Consequently $u^{k+1} \equiv \bar{u}^{k+1}>0$ on $U_{k}$. Since $U_{k+1}=\left\{u^{k+1}>0\right\}$, (4.16) follows.

3. Next we prove

$$
\left|\partial U_{k+1}\right|=0 \text {. }
$$


To confirm this claim, first define

$$
\tilde{u}^{k+1}(x):= \begin{cases}u^{k+1}(x) & \left(x \in \bar{U}_{k}\right) \\ \max _{y \in \bar{U}_{k}}\left\{u^{k+1}(y)-|x-y|, 0\right\} & \left(x \in \mathbb{R}^{2}-\bar{U}_{k}\right) .\end{cases}
$$

Reasoning as above, we conclude that $u^{k+1} \equiv \tilde{u}^{k+1}$, and in particular that $U_{k+1}$ is bounded.

As we know by induction that $\left|\partial U_{k}\right|=0$, we have

$$
\left|\partial U_{k+1} \cap \partial U_{k}\right|=0 .
$$

Set $E:=\partial U_{k+1}-\partial U_{k}$ and suppose $|E|>0$. Take $x \in E$ to be a point of density one for E:

$$
\lim _{r \rightarrow 0} \frac{|E \cap B(x, r)|}{|B(x, r)|}=1 .
$$

Since $x \in \partial U_{k+1}$ there exist points $\left\{x_{i}\right\}_{i=1}^{\infty} \subset U_{k+1}$, with $x_{i} \rightarrow x$. But recall $u^{k+1} \equiv \tilde{u}^{k+1}$. This means there exist points $\left\{y_{i}\right\}_{i=1}^{\infty} \subset \bar{U}_{k}$ with

$$
u^{k+1}\left(x_{i}\right)=u^{k+1}\left(y_{i}\right)-\left|x_{i}-y_{i}\right| \quad(i=1,2, \ldots) .
$$

We may assume $y_{i} \rightarrow y \in \bar{U}_{k}$. Then

$$
0=u^{k+1}(x)=u^{k+1}(y)-|x-y|,|x-y|>0 .
$$

Thus $u^{k+1}$ increases linearly, with slope 1 , on the line segment $[x, y]$. In particular

$$
\max _{\partial B(x, r)} u^{k+1}=r
$$

for small $r>0$.

But since $u^{k+1}=0$ on $E$ and $\left|D u^{k+1}\right| \leq 1$ a.e., (4.20) implies $\max _{B(x, r)} u^{k+1} \leq \frac{1}{2} r$ for small $r>0$. This is a contradiction to (4.21). So $\left|\partial U_{k+1}-\partial U_{k}\right|=0$ after all, and assertion (4.18) is proved.

4. Next we demonstrate that (4.10) holds and that the pair $\left(u^{k+1}, w^{k+1}\right)$ solves

$$
\left\{\begin{array}{l}
w^{k+1}+h \partial I_{\infty}\left(u^{k+1}\right) \ni(1+h) w^{k} \\
u^{k+1} \in \beta\left(w^{k+1}\right)
\end{array}\right.
$$

where

$$
w^{k}:=\chi_{U_{k}}, w^{k+1}:=\chi_{U_{k+1}} .
$$

Firstly, since $w^{k+1}=\chi_{\left\{u^{k+1}>0\right\}} \in \alpha\left(u^{k+1}\right)$, we have $u^{k+1} \in \beta\left(w^{k+1}\right)$. 
Next, remember that $J_{k+1}\left[u^{k+1}\right] \leq J_{k+1}[\hat{v}]$ for all $\hat{v}$ with $|D \hat{v}| \leq 1$ a.e.; which is to say,

$$
\int_{\mathbb{R}^{2}} u^{k+1} d x-(1+h) \int_{U_{k}} u^{k+1} d x \leq \int_{\mathbb{R}^{2}} \hat{v}^{+} d x-(1+h) \int_{U_{k}} \hat{v} d x
$$

for all such $\hat{v}$.

Now take $\tau<0$ and set $\hat{v}:=u^{k+1}+\tau$ in (4.23). Then

$$
i(\tau):=\int_{\mathbb{R}^{2}}\left(u^{k+1}+\tau\right)^{+} d x-(1+h) \int_{U_{k}} u^{k+1}+\tau d x
$$

has a minimum at $\tau=0$. We compute:

$$
0 \geq \lim _{\tau \rightarrow 0^{-}} \frac{i(\tau)-i(0)}{\tau}=\left|U_{k+1}\right|-(1+h)\left|U_{k}\right|
$$

Now let $\tau>0$, and put $\hat{v}:=u^{k+1}+\tau+\phi$ in (4.23), where $\phi$ is smooth, $\phi \equiv 0$ on $\bar{U}_{k+1}$, $\phi<0$ on $\mathbb{R}^{2}-\bar{U}_{k+1}, \phi \equiv-1$ outside some large disk, $|D \phi| \leq 1$. Then

$$
\begin{aligned}
0 & \leq \int_{\mathbb{R}^{2}} \frac{\left(u^{k+1}+\tau+\phi\right)^{+}-u^{k+1}}{\tau} d x-(1+h)\left|U_{k}\right| \\
& =\left|\bar{U}_{k+1}\right|-(1+h)\left|U_{k}\right|+\int_{\mathbb{R}^{2}-U_{k+1}} \frac{(\tau+\phi)^{+}}{\tau} d x
\end{aligned}
$$

Let $\tau \rightarrow 0^{+}$and recall $\left|\partial U_{k+1}\right|=0$, to deduce

$$
0 \leq\left|U_{k+1}\right|-(1+h)\left|U_{k}\right|
$$

This proves (4.10).

Next, take any Lipschitz function $v$ such $|D v| \leq 1$ and $v \leq 0$. Select $0<\lambda<1$ and put $\hat{v}:=(1-\lambda) u^{k+1}+\lambda v$ in (4.23). Then

$$
(1+h) \int_{U_{k}} v-u^{k+1} d x \leq \int_{\mathbb{R}^{2}} \frac{\left(u^{k+1}+\lambda\left(v-u^{k+1}\right)\right)^{+}-u^{k+1} d x}{\lambda} .
$$

Taking the limit as $\lambda \rightarrow 0$ yields

$$
(1+h) \int_{U_{k}} v-u^{k+1} d x \leq \int_{U_{k+1}} v-u^{k+1} d x
$$

This inequality holds if $v \leq 0$. Given then any bounded $v$ with $|D v| \leq 1$, replace $v$ by $v-c$ in $(4.25)$, for $c:=\max _{\mathbb{R}^{n}} v$. Then

$$
(1+h) \int_{U_{k}} v-c-u^{k+1} d x \leq \int_{U_{k+1}} v-c-u^{k+1} d x
$$


Since $\left|U_{k+1}\right|=(1+h)\left|U_{k}\right|$, we conclude:

$$
(1+h) \int_{U_{k}} v-u^{k+1} d x \leq \int_{U_{k+1}} v-u^{k+1} d x
$$

that is,

$$
\int_{\mathbb{R}^{2}}\left((1+h) w^{k}-w^{k+1}\right)\left(v-u^{k+1}\right) d x \leq 0
$$

for all bounded $v$ with $|D v| \leq 1$. Since we can if necessary redefine $v$ off $U_{k+1}$, the inequality (4.26) is valid for each function $v$ with $|D v| \leq 1$. But this means precisely that

$$
(1+h) w^{k}-w^{k+1} \in \partial I_{\infty}\left[u^{k+1}\right]=h \partial I_{\infty}\left[u^{k+1}\right] .
$$

5. Assume now that $\hat{u}^{k}, \hat{w}^{k}, \hat{U}_{k}(k=1, \ldots)$ comprise another solution, corresponding to a different initial set $\hat{U}_{0}$. We establish next a comparison result, namely that

$$
\hat{U}_{k} \supset \supset U_{k} \quad \text { implies } \quad \hat{U}_{k+1} \supset \supset U_{k+1} \quad(k=0,1, \ldots) .
$$

(The notation " $U \supset \supset$ " means $U \supset \bar{W} \supset W$.)

To prove (4.27), define $J_{k+1}[\cdot]$ as above and set $\hat{J}_{k+1}[\hat{v}]:=\int_{\mathbb{R}^{2}} \hat{v}^{+}-(1+h) \hat{v} \hat{w}^{k} d x$ for $\hat{w}^{k}=\chi_{\hat{U}_{k}}$. So $u^{k+1}$ minimizes $J_{k+1}[\cdot], \hat{u}^{k+1}$ minimizes $\hat{J}_{k+1}[\cdot], U_{k+1}=\left\{u^{k+1}>0\right\}$, $\hat{U}_{k+1}=\left\{\hat{u}^{k+1}>0\right\}$.

Then

$$
\left\{\begin{aligned}
J_{k+1}\left[u^{k+1}\right] & \leq J_{k+1}\left[\min \left(u^{k+1}, \hat{u}^{k+1}\right)\right] \\
\hat{J}_{k+1}\left[\hat{u}^{k+1}\right] & \leq \hat{J}_{k+1}\left[\max \left(u^{k+1}, \hat{u}^{k+1}\right)\right]
\end{aligned}\right.
$$

The first inequality implies

$$
\int_{\left\{u^{k+1}>\hat{u}^{k+1}\right\}} u^{k+1}-(1+h) u^{k+1} w^{k} d x \leq \int_{\left\{u^{k+1}>\hat{u}^{k+1}\right\}} \hat{u}^{k+1}-(1+h) \hat{u}^{k+1} w^{k} d x
$$

and thus

$$
\int_{\mathbb{R}^{2}}\left(u^{k+1}-\hat{u}^{k+1}\right)^{+} d x \leq(1+h) \int_{\mathbb{R}^{2}} w^{k}\left(u^{k+1}-\hat{u}^{k+1}\right)^{+} d x .
$$

Likewise the second line of (4.28) says

$$
\int_{\left\{u^{k+1}>\hat{u}^{k+1}\right\}} \hat{u}^{k+1}-(1+h) \hat{u}^{k+1} \hat{w}^{k} d x \leq \int_{\left\{u^{k+1}>\hat{u}^{k+1}\right\}} u^{k+1}-(1+h) u^{k+1} \hat{w}^{k} d x
$$

whence

$$
(1+h) \int_{\mathbb{R}^{2}}\left(u^{k+1}-\hat{u}^{k+1}\right)^{+} \hat{w}^{k} d x \leq \int_{\mathbb{R}^{2}}\left(u^{k+1}-\hat{u}^{k+1}\right)^{+} d x .
$$


This inequality and (4.29) imply

$$
\int_{\mathbb{R}^{2}}\left(\hat{w}^{k}-w^{k}\right)\left(u^{k+1}-\hat{u}^{k+1}\right)^{+} d x \leq 0
$$

That is,

$$
\int_{\hat{U}_{k}-U_{k}}\left(u^{k+1}-\hat{u}^{k+1}\right)^{+} d x \leq 0,
$$

since $\hat{U}_{k} \supset U_{k}$. Consequently

$$
u^{k+1} \leq \hat{u}^{k+1} \quad \text { on } \hat{U}_{k}-U_{k}
$$

Take now any point $x \in U_{k+1}-U_{k}$ and write $\delta:=u^{k+1}(x)>0$. Since $u^{k+1}=\tilde{u}^{k+1}$, there exists a point $y \in \bar{U}_{k}$ such that $u^{k+1}$ increases at rate one along the closed segment $S=[x, y]$.

Observe that since $\hat{U}_{k} \supset \supset U_{k}$, there exists a point $z$ lying on the open segment $(x, y)$ with $z \in \hat{U}_{k}-U_{k}$. Let $r=|x-z|>0$. Then, since $u^{k+1}$ increases at rate one along $S$,

$$
u^{k+1}(z)=\delta+r
$$

Thus (4.31) implies

$$
\hat{u}^{k+1}(z) \geq \delta+r
$$

Since $\left|D \hat{u}^{k+1}\right| \leq 1$ a.e., it follows that

$$
B^{0}(z, \delta+r) \subset \hat{U}^{k+1}
$$

Thus

$$
B^{0}(x, \delta) \subset \hat{U}^{k+1}
$$

So each point in $U_{k+1}-U_{k}$ is the center of a ball lying in $\hat{U}^{k+1}$. The same is true for each point in $U_{k}$, since $U_{k} \subset \hat{U}_{k} \subseteq \hat{U}_{k+1}$. Thus $U_{k+1} \subseteq \hat{U}_{k+1}$.

Since $\hat{U}_{k} \supset \supset U_{k}$, the same conclusion holds with $U_{k}$ replaced by a small translation in any direction. Thus a small translation of $\hat{U}_{k+1}$ in any direction likewise lies within $\hat{U}_{k+1}$, and so assertion (4.27) follows.

6. Suppose now that $U_{k} \subset B^{0}(0, R) \subset \subset B^{0}(0, R+\varepsilon)=: \hat{U}_{k}$. By symmetry and (4.24) (with $\hat{U}_{k}, \hat{U}_{k+1}$ replacing $\left.U_{k}, U_{k+1}\right)$, we see that $\hat{U}_{k+1}=B^{0}\left(0,(1+h)^{1 / 2}(R+\varepsilon)\right.$ ). But (4.27) says

$$
U_{k+1} \subseteq \hat{U}_{k+1}
$$

This containment holds for all $\varepsilon>0$, and assertion (iv) of the theorem follows. 
7. It remains to show uniqueness of the pair $\left(u^{k+1}, w^{k+1}\right)$. So suppose $w^{k+1}=\chi_{U_{k+1}}$ as above, and also

$$
\hat{w}^{k+1}+h \partial I_{\infty}\left[\hat{u}^{k+1}\right] \ni(1+h) w^{k}
$$

for $\hat{U}_{k+1}=\left\{\hat{u}^{k+1}>0\right\}, \hat{w}^{k+1}=\chi_{\hat{U}_{k+1}}$. Suppose $|D v| \leq 1$. Then (4.32) implies

$$
\int_{\mathbb{R}^{2}}\left((1+h) w^{k}-\hat{w}^{k+1}\right)\left(v-\hat{u}^{k+1}\right) d x \leq 0,
$$

and thus

$$
\int_{\mathbb{R}^{2}}\left(\hat{u}^{k+1}\right)^{+}-(1+h) \hat{u}^{k+1} w^{k} d x \leq \int_{\mathbb{R}^{n}} v^{+}-(1+h) v w^{k} d x,
$$

since $\hat{w}^{k+1} \hat{u}^{k+1}=\left(\hat{u}^{k+1}\right)^{+}$and $\hat{w}^{k+1} v \leq v^{+}$. So $\hat{u}^{k+1}$ minimizes $J_{k+1}[\cdot]$. But also

$$
J_{k+1}\left[\frac{u^{k+1}+\hat{u}^{k+1}}{2}\right] \leq \frac{1}{2} J_{k+1}\left[u^{k+1}\right]+\frac{1}{2} J_{k+1}\left[\hat{u}^{k+1}\right] .
$$

Consequently $\frac{u^{k+1}+\hat{u}^{k+1}}{2}$ is also a minimizer. But then we deduce as before that

$$
\left|D\left(\frac{u^{k+1}+\hat{u}^{k+1}}{2}\right)\right|=1 \text { a.e. on }\left\{\frac{u^{k+1}+\hat{u}^{k+1}}{2}>0\right\}=U_{k+1} \cup \hat{U}_{k+1} \text {. }
$$

Since $\left|D u^{k+1}\right| \leq 1,\left|D \hat{u}^{k+1}\right| \leq 1$ a.e., this implies

$$
D u^{k+1}=D \hat{u}^{k+1} \text { a.e. in } \mathbb{R}^{2} .
$$

As $u^{k+1}, \hat{u}^{k+1}$ both vanish outside some large ball, we deduce that $u^{k+1} \equiv \hat{u}^{k+1}, U_{k+1}=$ $\hat{U}_{k+1}$, and therefore $w^{k+1} \equiv \hat{w}^{k+1}$.

Remark. We note also

$$
u^{k+1} \geq u^{k} \quad(k=1, \ldots) .
$$

To see this, observe first that (4.31) implies

$$
u^{k+1} \geq u^{k} \quad \text { on } U_{k}-U_{k-1} .
$$

Now let $\hat{w}^{k}=\chi_{U_{k-1}}, \hat{u}^{k+1}=u^{k}$. Then (4.29), (4.30) (with the roles of $u^{k+1}, \hat{u}^{k+1}$ and of $w^{k}, \hat{w}^{k}$ reversed) give:

$$
\left\{\begin{array}{c}
\int_{\mathbb{R}^{n}}\left(\hat{u}^{k+1}-u^{k+1}\right)^{+} d x \leq(1+h) \int_{\mathbb{R}^{2}} \hat{w}^{k}\left(\hat{u}^{k+1}-u^{k+1}\right)^{+} d x \\
(1+h) \int\left(\hat{u}^{k+1}-u^{k+1}\right)^{+} w^{k} d x \leq \int_{\mathbb{R}^{2}}\left(\hat{u}^{k+1}-u^{k+1}\right)^{+} d x . \\
17
\end{array}\right.
$$


Combining, we deduce

$$
\int_{\mathbb{R}^{2}}\left(\hat{u}^{k+1}-u^{k+1}\right)^{+}\left(w^{k}-\hat{w}^{k}\right) d x \leq 0
$$

Since $w^{k}=\chi_{U_{k}} \geq \hat{w}^{k}=\chi_{U_{k-1}}$, the integrand is in fact nonnegative. Thus we must have had equality above; that is,

$$
\int_{\mathbb{R}^{n}}\left(\hat{u}^{k+1}-u^{k+1}\right)^{+} d x=(1+h) \int_{\mathbb{R}^{n}} \hat{w}^{k}\left(\hat{u}^{k+1}-u^{k+1}\right)^{+} d x .
$$

Rewriting, we deduce

$$
\int_{\mathbb{R}^{2}}\left(u^{k}-u^{k+1}\right)^{+} d x=(1+h) \int_{U_{k-1}}\left(u^{k}-u^{k+1}\right)^{+} d x
$$

and so

$$
\int_{\mathbb{R}^{2}-U_{k-1}}\left(u^{k}-u^{k+1}\right)^{+} d x=h \int_{U_{k-1}}\left(u^{k}-u^{k+1}\right)^{+} d x .
$$

But (4.34) says the term on the left is zero. Consequently $u^{k+1} \geq u^{k}$ on $U_{k-1}$. This implies (4.33).

Remark. Observe also from (4.8), (4.10) that

$$
\left\|\frac{w^{k+1}-w^{k}}{h}\right\|_{L^{1}\left(\mathbb{R}^{2}\right)}=\left\|w^{k}\right\|_{L^{1}\left(\mathbb{R}^{2}\right)} \quad(k=0, \ldots) .
$$

Next recall that if $E$ is a measurable subset of $\mathbb{R}^{n}$, the perimeter of $E$ is

$$
\operatorname{Per}(E):=\sup _{|\boldsymbol{\phi}| \leq 1}\left\{\int_{E} \operatorname{div} \phi d x\right\}
$$

the supremum taken over $C^{1}$ vector fields $\boldsymbol{\phi}$ with compact support, such that $|\boldsymbol{\phi}| \leq 1$ on $\mathbb{R}^{n}$. Let us hereafter assume:

$$
\operatorname{Per}\left(U_{0}\right)<\infty .
$$

Proposition 4.2. For $k=0,1, \ldots$

$$
\operatorname{Per}\left(U_{k+1}\right) \leq(1+h) \operatorname{Per}\left(U_{k}\right) .
$$

Proof. 1. Assume first $f$ is smooth, with compact support, $2<p<\infty$, and the pair $u, w$ solve

$$
\left\{\begin{array}{l}
w-\operatorname{div}\left(|D u|^{p-2} D u\right)=f \quad \text { in } \mathbb{R}^{2} \\
u=\beta_{\varepsilon}(w)
\end{array}\right.
$$


where $\beta_{\varepsilon}: \mathbb{R} \rightarrow \mathbb{R}$ is a smooth, strictly increasing function approximating $\beta$.

Assuming $u, w$ are smooth, we differentiate the PDE (4.37) with respect to $x_{k}$ :

$$
w_{x_{k}}-\left(|D u|^{p-2} u_{x_{k} x_{i}}+(p-2)|D u|^{p-4} u_{x_{j}} u_{x_{i}} u_{x_{k} x_{j}}\right)_{x_{i}}=f_{x_{k}} .
$$

Take $\delta>0$, multiply $(4.38)$ by $\frac{u_{x_{k}}}{\left(|D u|^{2}+\delta^{2}\right)^{1 / 2}}$, and integrate:

$$
\begin{aligned}
& \int_{\mathbb{R}^{2}} \frac{D w \cdot D u}{\left(|D u|^{2}+\delta^{2}\right)^{1 / 2}} d x \\
& +\int_{\mathbb{R}^{2}}\left(|D u|^{p-2} u_{x_{k} x_{i}}+(p-2)|D u|^{p-4} u_{x_{i}} u_{x_{j}} u_{x_{k} x_{j}}\right)\left(\frac{u_{x_{k}}}{\left(|D u|^{2}+\delta^{2}\right)^{1 / 2}}\right)_{x_{i}} d x \\
& \quad=\int_{\mathbb{R}^{2}} \frac{D f \cdot D u}{\left(|D u|^{2}+\delta^{2}\right)^{1 / 2}} d x
\end{aligned}
$$

Writing out $\left(\frac{u_{x_{k}}}{\left(|D u|^{2}+\delta^{2}\right)^{1 / 2}}\right)_{x_{i}}$, we note that the integrand of the second term on the left is pointwise nonnegative. Thus

$$
\int_{\mathbb{R}^{2}} \frac{D w \cdot D u}{\left(|D u|^{2}+\delta^{2}\right)^{1 / 2}} d x \leq \int_{\mathbb{R}^{2}}|D f| d x
$$

Let $\delta \rightarrow 0$ :

$$
\int_{\{D u \neq 0\}} \frac{D w \cdot D u}{|D u|} d x \leq \int_{\mathbb{R}^{2}}|D f| d x .
$$

But since $u=\beta_{\varepsilon}(w)$ and $\beta_{\varepsilon}^{\prime}>0$, it follows that

$$
\int_{\mathbb{R}^{2}}|D w| d x \leq \int_{\mathbb{R}^{2}}|D f| d x .
$$

2. Next take $f=f_{\varepsilon}$ to be a smooth approximation to $(1+h) w^{k}=(1+h) \chi_{U_{k}}$, with

$$
\left\{\begin{array}{l}
0 \leq f_{\varepsilon} \leq 1, f_{\varepsilon} \rightarrow(1+h) \chi_{U_{k}} \text { in } L^{1} \\
\int_{\mathbb{R}^{2}}|D f| d x \leq \operatorname{Per}\left(U_{k}\right)(1+h)(1+\varepsilon)
\end{array}\right.
$$

cf. [E-G2, p. 172]. We fix $p<\infty$ and solve (4.37). Then (4.39) implies

$$
\int_{\mathbb{R}^{2}}|D w| d x \leq(1+\varepsilon)(1+h) \operatorname{Per}\left(U_{k}\right)
$$

3. Now write $w=w_{\varepsilon, p}$ to display the dependence of the solution of (4.37) on $\varepsilon, p$. Thus

$$
\int_{\mathbb{R}^{2}}\left|D w_{\varepsilon, \delta}\right| d x \leq(1+\varepsilon)(1+h) \operatorname{Per}\left(U_{k}\right) .
$$


Then maximum principle implies

$$
0 \leq w_{\varepsilon, \delta} \leq 1
$$

Owing to (4.40), (4.41) $\left\{w_{\varepsilon, p}\right\}_{0<\varepsilon \leq 1}^{n \leq p<\infty}$ is bounded in $W_{\text {loc }}^{1,1}\left(\mathbb{R}^{2}\right)$; and so

$$
w_{\varepsilon, p_{k}} \rightarrow w_{\varepsilon} \quad \text { in } L_{\mathrm{loc}}^{1}\left(\mathbb{R}^{2}\right)
$$

for some sequence $p_{k} \rightarrow \infty$. We have

$$
\left\{\begin{array}{l}
B V\left(w_{\varepsilon}\right) \leq(1+\varepsilon)(1+h) \operatorname{Per}\left(U_{k}\right), \\
0 \leq w_{\varepsilon} \leq 1 .
\end{array}\right.
$$

Here $B V$ denotes the bounded variation seminorm. From (4.37) it follows that

$$
\left\{\begin{array}{l}
f_{\varepsilon}-w_{\varepsilon} \in \partial I_{\infty}\left[u_{\varepsilon}\right] \quad \text { in } \mathbb{R}^{2} \\
u_{\varepsilon}=\beta_{\varepsilon}\left(w_{\varepsilon}\right) .
\end{array}\right.
$$

Now from the uniform bounds (4.42) we can select a sequence $\varepsilon_{j} \rightarrow 0$ such that

$$
w_{\varepsilon_{j}} \rightarrow w \text { in } L_{\mathrm{loc}}^{1}\left(\mathbb{R}^{2}\right)
$$

where

$$
B V(w) \leq(1+h) \operatorname{Per}\left(U_{k}\right), 0 \leq w \leq 1 .
$$

But $\left|D u_{\varepsilon}\right| \leq 1$ a.e., and so we may also assume $u_{\varepsilon_{j}} \rightarrow u$ uniformly. Then

$$
u \in \beta(w), w \in \alpha(u) .
$$

As (4.43) says

$$
\int_{\mathbb{R}^{2}}\left(f_{\varepsilon}-w_{\varepsilon}\right)\left(v-u_{\varepsilon}\right) d x \leq 0
$$

for all $v$ with $|D v| \leq 1$ a.e., we deduce

$$
\int_{\mathbb{R}^{2}}(f-w)(v-u) d x \leq 0
$$

for all such $v$, where $f=(1+h) \chi_{U_{k}}$. Thus

$$
\left\{\begin{array}{l}
w+h \partial I_{\infty}[u] \ni(1+h) w^{k} \\
u \in \beta(w) .
\end{array}\right.
$$

This in turn implies that $u$ minimizes

$$
J_{k+1}[v]=\int_{\mathbb{R}^{2}} v^{+}-(1+h) v w^{k} d x .
$$

Thus, by uniqueness of minimizers, $u=u^{k+1}$. Now $w \in \alpha(u)$ implies $w \equiv 1$ on $U_{k+1}$. Additionally (4.44) implies $w \equiv 0$ on $\mathbb{R}^{2}-\bar{U}_{k+1}$. Since $\left|\partial U_{k+1}\right|=0$, we deduce

$$
w=\chi_{U_{k+1}} \text { a.e. }
$$

Therefore

$$
\operatorname{Per}\left(U_{k+1}\right)=B V(w) \leq(1+h) \operatorname{Per}\left(U_{k}\right) .
$$

Assume now we take a different starting set $\hat{U}_{0}$, and define $\hat{U}_{k}(k=1, \ldots)$ as above. 
Proposition 4.3. We have

$$
\left|\hat{U}_{k+1}-U_{k+1}\right| \leq(1+h)\left|\hat{U}_{k}-U_{k}\right| \quad(k=0, \ldots) .
$$

Proof. Consider in addition to the PDE (4.37) the PDE

$$
\left\{\begin{array}{l}
\hat{w}-\operatorname{div}\left(|D \hat{u}|^{p-2} D \hat{u}\right)=\hat{f} \quad \text { in } \mathbb{R}^{2} \\
\hat{u}=\beta_{\varepsilon}(\hat{w}) .
\end{array}\right.
$$

Let $\gamma: \mathbb{R} \rightarrow \mathbb{R}$ be smooth, with $0 \leq \gamma \leq 1, \gamma^{\prime} \geq 0$. Then from (4.37), (4.47) we deduce

$$
\begin{aligned}
\int_{\mathbb{R}^{2}}(\hat{w}-w) \gamma(\hat{u}-u)+\gamma^{\prime}(\hat{u}-u)\left(|D \hat{u}|^{p-2} D \hat{u}\right. & \left.-|D u|^{p-2} D u\right) \cdot D(\hat{u}-u) d x \\
& =\int_{\mathbb{R}^{2}}(\hat{f}-f) \gamma(\hat{u}-u) d x
\end{aligned}
$$

The second term inside the integral on the left is nonnegative and can be discarded. After approximation we thereby obtain:

$$
\int_{\mathbb{R}^{2}}(\hat{w}-w) \operatorname{sgn}_{+}(\hat{u}-u) d x \leq \int_{\mathbb{R}^{2}}(\hat{f}-f) \operatorname{sgn}_{+}(u-\hat{u}) d x
$$

for

$$
\operatorname{sgn}_{+}(r):= \begin{cases}1 & \text { if } r>0 \\ 0 & \text { if } r \leq 0\end{cases}
$$

Since $\beta_{\varepsilon}$ is strictly increasing and $u=\beta_{\varepsilon}(w), \hat{u}=\beta_{\varepsilon}(\hat{w})$, we see that

$$
\operatorname{sgn}_{+}(\hat{u}-u)=\operatorname{sgn}_{+}(\hat{w}-w) .
$$

Thus

$$
\begin{aligned}
\int_{\mathbb{R}^{2}}(\hat{w}-w)^{+} d x & \leq \int_{\mathbb{R}^{2}}(\hat{f}-f) \operatorname{sgn}_{+}(\hat{w}-w) d x \\
& \leq \int_{\mathbb{R}^{n}}(\hat{f}-f)^{+} d x .
\end{aligned}
$$

Letting $p \rightarrow \infty$ and then $\varepsilon \rightarrow 0$ as in the previous proof, we obtain estimate (4.46).

Remark. A similar proof shows

$$
\left|\hat{U}_{k+1} \triangle U_{k+1}\right| \leq(1+h)\left|\hat{U}_{k} \triangle U_{k}\right| \quad(k=0, \ldots)
$$

for the symmetric difference

$$
A \triangle B:=(A-B) \cup(B-A) .
$$

To see this, take $\gamma$ above to be a smooth approximation of

$$
\operatorname{sgn}(r):= \begin{cases}1 & \text { if } r>0 \\ 0 & \text { if } r=0 \\ -1 & \text { if } r<0\end{cases}
$$




\section{Weak solutions.}

In this section we prove that the approximations (4.3), (4.4) converge as $h \rightarrow 0$ to a weak solution of (4.1), (4.2).

Theorem 5.1. There exists a pair $(u, w)$ solving (4.1), (4.2) in the sense that:

(i) $w \in C^{0,1}\left([0, \infty) ; L^{1}\left(\mathbb{R}^{2}\right)\right), w(0)=\chi_{U_{0}}$.

(ii) $w_{t}$ is a nonnegative Radon measure.

(iii) $w-w_{t} \in \partial I_{\infty}[u]$ for a.e. $t>0$.

(iv) $\operatorname{spt}\left(w_{t}\right)=\partial U_{t}=\Gamma_{t}$ for a.e. $t>0$, where $U_{t}:=\{u(\cdot, t)>0\}$.

(v) $u=\operatorname{dist}\left(x, \Gamma_{t}\right)\left(x \in U_{t}, t \geq 0\right)$.

(vi) $w=\chi_{U_{t}}$ for a.e. $t \geq 0$.

(vii) $\left|\hat{U}_{t} \triangle U_{t}\right| \leq e^{t}\left|\hat{U}_{0} \triangle U_{0}\right|(t \geq 0)$ for any other solution built by the algorithm in $\S 4$.

Proof. 1. Define for $h>0$ :

$$
\begin{cases}w^{h}(x, t):=w^{k}(x) & \text { if } \quad x \in \mathbb{R}^{2}, k h \leq t<(k+1) h \\ u^{h}(x, t):=u^{k}(x) & \text { if } \quad x \in \mathbb{R}^{2}, k h \leq t<(k+1) h,\end{cases}
$$

$(k=1, \ldots)$. According to estimates (4.35), (4.36) $\left\{w^{h}\right\}_{0<h \leq 1}$ is bounded in $B V\left(\mathbb{R}^{2} \times[0, T]\right)$ for each $T>0$. Thus there exists a function $w \in B V\left(\mathbb{R}^{2} \times[0, T]\right)$ and a sequence $h_{j} \rightarrow 0$ such that

$$
w^{h_{j}} \rightarrow w \quad \text { in } L^{1}\left(\mathbb{R}^{2} \times[0, T]\right), \quad w^{h_{j}} \rightarrow w \quad \text { a.e. }
$$

for each $T>0$. In addition, since $U_{k+1} \supseteq U_{k}(k=0, \ldots)$ and $w^{k}=\chi_{U_{k}}$, we have $w_{t}^{h} \geq 0$ in the sense of distributions. Consequently $w_{t}^{h}$ is a nonnegative Radon measure. Hence, passing as necessary to a subsequence and relabelling, we have

$$
w_{t}^{h_{j}} \rightarrow \mu \text { weakly as measures, }
$$

where $\mu$ is a nonnegative Radon measure on $\mathbb{R}^{2} \times[0, \infty)$. Also estimate (4.35) implies

$$
\left\{\begin{array}{l}
\text { the mapping } t \mapsto w(\cdot, t) \text { is Lipschitz continuous } \\
\text { from }[0, T] \text { into } L^{1}\left(\mathbb{R}^{2}\right)
\end{array}\right.
$$

for each $T>0$. For each continuous function $f$, we can therefore write

$$
\int_{0}^{T} \int_{\mathbb{R}^{2}} f d \mu=\int_{0}^{T} \int_{\mathbb{R}^{2}} f d w_{t} d t
$$

where $w_{t}$ is for a.e. time $t$ a nonnegative Radon measure on $\mathbb{R}^{2}$. 
Likewise, $u^{k+1} \geq u^{k}(k=1, \ldots)$ according to (4.33) and so $u_{t}^{h}$ is a nonnegative Radon measure. Furthermore $\left|D u^{k}\right| \leq 1$ a.e., $D$ denoting here and later the gradient in the variable $x \in \mathbb{R}^{2}$. Consequently $\left\{u^{h}\right\}_{0<h \leq 1}$ is bounded in $B V\left(\mathbb{R}^{2} \times[0, T]\right)$ for each $T>0$; and thus, upon passing if necessary to yet a further subsequence and reindexing, we deduce

$$
\begin{cases}u^{h_{j}} \rightarrow u & \text { in } L^{1}\left(\mathbb{R}^{2} \times[0, T]\right) \\ u^{h_{j}} \rightarrow u & \text { uniformly, for a.e. } t>0\end{cases}
$$

for each $T>0$. In addition, $|D u| \leq 1$ a.e.

Furthermore, we have

$$
u_{t}^{h_{j}} \rightarrow \nu \text { weakly as measures, }
$$

where $\nu$ is a nonnegative Radon measure on $\mathbb{R}^{2} \times[0, \infty)$. In particular,

$$
\left\{\begin{array}{l}
\text { the mapping } t \mapsto u(\cdot, t) \text { is continuous from } \\
{[0, \infty) \text { into } C\left(\mathbb{R}^{2}\right), \text { for all but at }} \\
\text { most countably many times } J=\left\{t_{k}\right\}_{k=1}^{N},
\end{array}\right.
$$

$N \leq \infty$. This is so since $\nu\left(\mathbb{R}^{2} \times\{t=s\}\right)=0$ for all but at most countably many times $s$. We can define then for each "jump" time $t_{k} \in J$,

$$
u\left(\cdot, t_{k}\right):=\lim _{\substack{t \rightarrow t_{k}^{-} \\ t \notin J}} u(\cdot, t) .
$$

Then $u$ is defined now for all times $t>0$, and $|D u| \leq 1$ a.e. (We will see from Example A of $\S 7$ that the mapping $t \mapsto u$ need not be continuous when "a hole disappears".)

2. We assert

$$
w-w_{t} \in \partial I_{\infty}[u] \text { for a.e. } t \text {. }
$$

This means that

$$
\int_{\mathbb{R}^{2}} w(v-u(\cdot, t)) d x \leq \int_{\mathbb{R}^{2}}(v-u(\cdot, t)) d w_{t}
$$

for each $v$ with $|D v| \leq 1$. To prove this, recall from (4.22) that

$$
\int_{\mathbb{R}^{2}} w^{k}\left(\tilde{v}^{k+1}-u^{k+1}\right) d x \leq \int_{\mathbb{R}^{2}}\left(\frac{w^{k+1}-w^{k}}{h}\right)\left(\tilde{v}^{k+1}-u^{k+1}\right) d x
$$

if $\left|D \tilde{v}^{k+1}\right| \leq 1$ for $k=0,1,2, \ldots$. Take $\phi:[0, \infty) \rightarrow \mathbb{R}$ to be smooth, with compact support, fix $v: \mathbb{R}^{2} \rightarrow \mathbb{R}$ with $|D v| \leq 1$ a.e., and set $\tilde{v}^{k+1}=\phi((k+1) h) v$ in (5.11). Multiply by $h$ and sum:

$$
\int_{0}^{T} \int_{\mathbb{R}^{2}} w^{h}\left(\tilde{v}^{h}-u^{h}\right) d x \leq \int_{03}^{T} \int_{\mathbb{R}^{2}} w_{t}^{h}\left(\tilde{v}^{h}-u^{h}\right) d x d t .
$$


Here $\tilde{v}^{h}=\tilde{v}^{k}$ if $k h \leq t<(k+1) h$. According to (5.2), (5.5):

$$
\int_{0}^{T} \int_{\mathbb{R}^{2}} w^{h_{j}}\left(\tilde{v}^{h_{j}}-u^{h_{j}}\right) d x d t \rightarrow \int_{0}^{T} \int_{\mathbb{R}^{2}} w(\tilde{v}-u) d x d t
$$

where $\tilde{v}:=\phi v$. Additionally, (5.3) implies

$$
\int_{0}^{T} \int_{\mathbb{R}^{2}} w_{t}^{h_{j}} \tilde{v}^{h_{j}} d x d t \rightarrow \int_{0}^{T} \int_{\mathbb{R}^{2}} \tilde{v} d \mu
$$

It remains to analyze the limiting behavior of the term

$$
\begin{aligned}
\int_{0}^{T} \int_{\mathbb{R}^{2}} w_{t}^{h_{j}} u^{h_{j}} d x d t & =\int_{0}^{T} \int_{\mathbb{R}^{2}} w_{t}^{h_{j}} u d x d t \\
& \quad+\int_{0}^{T} \int_{\mathbb{R}^{2}} w_{t}^{h_{j}}\left(u^{h_{j}}-u\right) d x d t \\
= & : A_{1}+A_{2} .
\end{aligned}
$$

Estimate of $A_{1}$ : We have

$$
A_{1}=\int_{0}^{T} \int_{\mathbb{R}^{2}} w_{t}^{h_{j}}(u-\bar{u}) d x d t+\int_{0}^{T} \int_{\mathbb{R}^{2}} w_{t}^{h_{j}} \bar{u} d x d t
$$

for any function $\bar{u} \in C\left([0, T] \times \mathbb{R}^{2}\right)$ with compact support. Notice that

$$
\lim _{j \rightarrow \infty} \int_{0}^{T} \int_{\mathbb{R}^{2}} w_{t}^{h_{j}} \bar{u} d x d t=\int_{0}^{T} \int_{\mathbb{R}^{2}} \bar{u} d \mu
$$

Next fix $\varepsilon>0$. Then

$$
\limsup _{t \rightarrow t_{k}}\left\|u(\cdot, t)-u\left(\cdot, t_{k}\right)\right\|_{L^{\infty}\left(\mathbb{R}^{2}\right)} \geq \varepsilon
$$

for at most finitely many times $\left\{t_{k}\right\}_{k=1}^{M}, M=M(\varepsilon)$. Fix $\delta>0$ so small that

$$
2 M \delta \leq \varepsilon
$$

We assert next that we can construct $\bar{u} \in C\left([0, T] \times \mathbb{R}^{2}\right)$ so that

$$
\|\bar{u}(\cdot, t)-u(\cdot, t)\|_{L^{\infty}} \leq 3 \varepsilon
$$

provided

$$
t \notin \bigcup_{k=1}^{M}\left(t_{k}-\delta, t_{k}+\delta\right)=: \Delta
$$


To see this, observe that if $I$ denotes any of the closed subintervals making up $[0, T]-$ $\Delta$, then $\lim \sup _{t \rightarrow s}\|u(\cdot, t)-u(\cdot, s)\|_{L^{\infty}\left(\mathbb{R}^{2}\right)} \leq \varepsilon$ for each $s \in I$. It follows by a simple compactness argument that there must then exist $\delta>0$ with the property that for all $s, t \in I$, the inequality $|t-s| \leq \delta$ implies $\|u(\cdot, t)-u(\cdot, s)\|_{L^{\infty}\left(\mathbb{R}^{2}\right)} \leq 3 \varepsilon$. We subdivide $I$ into finitely many subintervals of length less than $\delta$, set $\bar{u}$ to equal $u$ at the endpoints of these subintervals, and extend $\bar{u}$ linearly. Since $u$ is nondecreasing in the variable $t$, we check that (5.18) follows.

Then

$$
\begin{aligned}
\left|\int_{0}^{T} \int_{\mathbb{R}^{2}} w_{t}^{h_{j}}(u-\bar{u}) d x d t\right| & \leq \varepsilon \int_{[0, T]-\Delta}\left\|w_{t}^{h_{j}}\right\|_{L^{1}} d t+C \int_{\Delta}\left\|w_{t}^{h_{j}}\right\|_{L^{1}} d t \\
& \leq C \varepsilon+C|\Delta| \leq C \varepsilon \quad \text { by }(4.35),(5.17) .
\end{aligned}
$$

Thus

$$
\limsup _{h_{j} \rightarrow 0}\left|A_{1}-\int_{0}^{T} \int_{\mathbb{R}^{2}} \bar{u} d \mu\right| \leq C \varepsilon
$$

Similarly

$$
\int_{0}^{T} \int_{\mathbb{R}^{2}} u-\bar{u} d \mu \leq C \varepsilon
$$

and so

$$
\lim _{h_{j} \rightarrow 0} A_{1}=\lim _{h_{j} \rightarrow 0} \int_{0}^{T} \int_{\mathbb{R}^{2}} w_{t}^{h_{j}} u d x d t=\int_{0}^{T} \int_{\mathbb{R}^{2}} u d \mu .
$$

Estimate of $A_{2}$ : We estimate using (4.35) that

$$
\left|\int_{0}^{T} \int_{\mathbb{R}^{2}} w_{t}^{h_{j}}\left(u^{h_{j}}-u\right) d x d t\right| \leq C \int_{0}^{T}\left\|u^{h_{j}}-u\right\|_{L^{\infty}} d t .
$$

But

$$
\left\|u^{h_{j}}-u\right\|_{L^{\infty}} \leq C\left\|u^{h_{j}}-u\right\|_{L^{1}}^{\frac{1}{3}}\left\|D u^{h_{j}}-D u\right\|_{L^{\infty}}^{\frac{2}{3}}
$$

for some constant $C$. Consequently

$$
\int_{0}^{T}\left\|u^{h_{j}}-u\right\|_{L^{\infty}} d t \leq C \int_{0}^{T}\left\|u^{h_{j}}-u\right\|_{L^{1}}^{\frac{1}{3}} d x \leq \varepsilon+C_{\varepsilon} \int_{0}^{T}\left\|u^{h_{j}}-u\right\|_{L^{1}} d t \rightarrow \varepsilon
$$

as $h_{j} \rightarrow 0$. Hence

$$
\lim _{h_{j} \rightarrow 0} A_{2}=\lim _{h_{j} \rightarrow 0} \int_{0}^{T} \int_{\mathbb{R}^{2}} w^{h_{j}}\left(u^{h_{j}}-u\right) d x d t=0 .
$$


Combining estimates (5.15), (5.19), (5.20), we deduce

$$
\int_{0}^{T} \int_{\mathbb{R}^{2}} w(\tilde{v}-u) d x d t \leq \int_{0}^{T} \int_{\mathbb{R}^{2}}(\tilde{v}-u) d \mu
$$

for $\tilde{v}=\phi v$. Thus for a.e. time $t$

$$
\int_{\mathbb{R}^{2}} w(v-u) d x \leq \int_{\mathbb{R}^{2}}(v-u) d w_{t}
$$

for all $v$ with $|D v| \leq 1$ a.e.. This proves (5.10), which is assertion (iii) of the Theorem.

3. Next define

$$
U_{t}:=\{u(\cdot, t)>0\}, \Gamma_{t}:=\partial U_{t}
$$

for $t \geq 0$. Since $|D u| \leq 1$ a.e., $U_{t}$ is open, and by construction $U_{t}$ is bounded.

We claim that for a.e. time $t>0$,

$$
\Gamma_{t}=\bigcap_{h>0} \bar{U}_{t+h}-U_{t}
$$

Indeed since $U_{t+h} \supseteq U_{t}, \Gamma_{t} \subseteq \bigcap_{h>0} \bar{U}_{t+h}-U_{t}$. Conversely, suppose $x \notin \bar{U}_{t}$. Then there exists a ball $B(x, r) \subset \mathbb{R}^{2}-\bar{U}_{t}$. By comparison, for some small $\delta>0$ we have $B(x, r / 2) \times\{t+h\} \subset \mathbb{R}^{2}-\bar{U}_{t+h}$ if $0<h \leq \delta$. That is, $x \notin \bar{U}_{t+h}$ for $0 \leq h \leq \delta$. Thus $x \in \bigcap_{h>0} \bar{U}_{t+h}$ implies $x \in \bar{U}_{t}$. In particular then $\bigcap_{h>0} \bar{U}_{t+h}-U_{t} \subseteq \bar{U}_{t}-U_{t}=\Gamma_{t}$. Assertion (5.21) is proved.

5. Note next that since $w^{h_{j}} \in\{0,1\}$ a.e. and $w^{h_{j}} \rightarrow w$ a.e., we likewise have $w \in\{0,1\}$ a.e.. In particular for a.e. $t$ :

$$
w=\chi_{E_{t}} \quad \text { a.e. in } \mathbb{R}^{2},
$$

where the set $E_{t}$ has finite perimeter, $U_{t} \subseteq E_{t} \subseteq \bar{U}_{t}$. Furthermore

$$
\left\{\begin{array}{l}
E_{s} \supseteq E_{t} \text { if } s>t, E_{0}=U_{0} \\
\text { and }\left|E_{t}\right|=e^{t}\left|E_{0}\right|
\end{array}\right.
$$

The last equality here follows from (4.35). In addition, we claim

$$
\operatorname{spt}\left(w_{t}\right) \subseteq \Gamma_{t} .
$$

To verify this, note that

$$
E_{t+h}-E_{t} \subseteq \bar{U}_{t+h}-U_{t},
$$

and (5.21) implies (5.24). 
6. Now since $w-w_{t} \in \partial I_{\infty}[u]$ for a.e. $t>0$, we have

$$
\max _{|D v| \leq 1}\left\{\int_{E_{t}} v d x-\int_{\Gamma_{t}} v d w_{t}\right\}=\int_{E_{t}} u(x, t) d x-\int_{\Gamma_{t}} u(x, t) d w_{t} .
$$

In particular since $u(\cdot, t)=0$ on $\Gamma_{t} \supseteq \operatorname{spt}\left(w_{t}\right)$, (5.25) implies

$$
u(x, t)=\operatorname{dist}\left(x, \operatorname{spt}\left(w_{t}\right)\right) \quad\left(x \in E_{t}\right) .
$$

But this in turn implies

$$
\operatorname{spt}\left(w_{t}\right)=\Gamma_{t}
$$

as otherwise $u$ defined by (5.26) would not equal 0 on all of $\Gamma_{t}$. Hence

$$
u(x, t)=\operatorname{dist}\left(x, \Gamma_{t}\right) \quad \text { on } E_{t}
$$

and, in particular,

$$
u(x, t)=\operatorname{dist}\left(x, \Gamma_{t}\right) \quad \text { on } U_{t} .
$$

7. We next assert

$$
\left|E_{t}-U_{t}\right|=0 \text { for a.e. } t>0 \text {, }
$$

and consequently

$$
w=\chi_{U_{t}} \text { a.e. in } \mathbb{R}^{2}
$$

We will confirm (5.29) by proving

$$
\left|U_{t}\right|=e^{t}\left|U_{0}\right| \quad(t \geq 0) .
$$

For this, we note first that (4.27) implies that

$$
\tilde{E}_{t} \subseteq E_{t} \quad \text { if } \quad \tilde{U}_{0} \subset \subset U_{0} .
$$

Suppose in particular $\tilde{U}_{0}$ is compactly contained within $U_{0}$. Then for fixed $\varepsilon>0$ sufficiently small, the $\varepsilon$-translate of $\tilde{U}_{0}$ in any direction also lies in $U_{0}$. Consequently the $\varepsilon$-translate of $\tilde{E}_{t}$ in any direction lies within $E_{t}$. Thus in fact $\tilde{E}_{t} \subset U_{t}$. But $\left|\tilde{E}_{t}\right|=e^{t}\left|\tilde{U}_{0}\right|$, and so $\left|U_{t}\right| \geq e^{t}\left|\tilde{U}_{0}\right|$ for each $\tilde{U}_{0}$ compactly contained in $U_{0}$. Hence

$$
\left|U_{t}\right| \geq e^{t}\left|U_{0}\right|
$$

But since $U_{t} \subseteq E_{t} \subseteq \bar{U}_{t}$ and $\left|E_{t}\right|=e^{t}\left|U_{0}\right|$, we deduce that $\left|U_{t}\right|=e^{t}\left|U_{0}\right|$. This proves (5.31), and so (5.29), (5.30).

8. Estimate (vii) follows from the Remark after Proposition 4.3.

Remark. Notice from the example of an outward pointing corner, discussed in $\S 7$.B following, that although $\operatorname{spt}\left(w_{t}\right)=\Gamma_{t}$, this does not mean that each point of $\Gamma_{t}$ is moving. 


\section{Geometric motion of the interface.}

Since $w=\chi_{U_{t}}$ and $u=\operatorname{dist}\left(\cdot, \Gamma_{t}\right)$ inside $U_{t}$, the entire evolution is determined by the geometric motion of the free boundary $\Gamma_{t}$ for $t \geq 0$. In this section we derive heuristically the law governing the changing shape of $\left\{\Gamma_{t}\right\}_{t \geq 0}$. We will adapt some terminology and methods from Bergwall [B1, B2] and [E-G1].

First, recall from $\S 3$ that we can formally interpret our evolution as saying

$$
\left\{\begin{array}{l}
w_{t}-\operatorname{div}(a D u)=w \\
u \in \beta(w)
\end{array} \quad \text { in } \mathbb{R}^{2} \times(0, T)\right.
$$

in some weak sense, where the function $a$ is the Lagrange multiplier for the constraint that $|D u| \leq 1$.

An ODE along transport rays. The idea is to turn attention at each fixed time $t \geq 0$ to the Lagrange multiplier $a$, which as we have seen in $\S 2$ is the material speed. Since

$$
-\operatorname{div}(a D u)=1 \quad \text { in } U_{t},
$$

we have, formally,

$$
-D a \cdot D u-a \Delta u=1 \text { in } U_{t} .
$$

We can as follows regard this identity as an ODE for $a$, for any line segment along which $u$ is linear, with slope 1 .

For definiteness suppose $y \in \Gamma_{t}$ and the outer normal to $\Gamma_{t}$ at $y$ is vertical. We wish to compute the outward normal velocity $V$ at $y$. According to (3.11), $V=a$ on $\Gamma_{t}$. Now suppose $\kappa$ is the curvature of $\Gamma_{t}$ at $y$ and $r=1 / \kappa$ is the radius of curvature (if $\kappa>0$ ). Assume the origin is at distance $r$ below $y$. Then $u=\operatorname{dist}\left(\cdot, \Gamma_{t}\right)$ will decrease at rate one along some linear segment starting at a point $x \in U_{t}$ and ending at $y$. We say that $x$ belongs to the ridge of $U_{t}$, which is the closure of the set on which the distance function is not differentiable. The distance $|x-y|$ is less than or equal to $r$. Let us now think of $x, y$ as lying in one dimension, and so $0 \leq x<y$.

Our intention is computing $V=a$ at the boundary point $y$. We will do so utilizing an ODE that we obtain upon writing $a^{\prime}$ for $-D a \cdot D u$ in (6.1). We thereby convert the PDE (6.1) into the ODE

$$
a^{\prime}-a \Delta u=1 \quad(x \leq s \leq y)
$$

holding along the ray connecting $y$ to the ridge point $x$.

We also need a boundary condition for the ODE, and for this we impose the condition

$$
a(x)=0 .
$$


The equality (6.3) is motivated by Monge-Kantorovich mass transfer theory, and in particular by the papers Janfalk [J1, J2] and Evans-Gangbo [E-G1].

The idea in the current context is that the material motion is directly towards the closest point on the boundary. So at a point $x$ belonging to the ridge, for which there are two or more closest points on the boundary, the velocity to be consistent must be zero.

But as $u=\operatorname{dist}\left(\cdot, \Gamma_{t}\right)$, we have $-\Delta u(s)=\frac{1}{s}$ : see, for instance, Gilbarg-Trudinger [G-T, page 354]. So our ODE reads

$$
\left\{\begin{array}{l}
a^{\prime}+\frac{a}{s}=1 \quad(x \leq s \leq y) \\
a(x)=0
\end{array}\right.
$$

Solving, we see

$$
a(s)=\frac{s}{2}-\frac{x^{2}}{2 s} \quad(x \leq s \leq y)
$$

and consequently

$$
V=a(y)=\frac{y}{2}-\frac{x^{2}}{2 y}
$$

But $y=r=\frac{1}{\kappa}, x=r-\gamma=\frac{1}{\kappa}-\gamma, \gamma$ denoting the distance along the ray from $y$ to the ridge. We hereafter refer to this length as the "ridge distance": see Bergwall [B1, B2] for more.

A nonlocal geometric evolution. Substituting above, we obtain the geometric law of interface motion:

$$
V=\gamma\left(1-\frac{\kappa \gamma}{2}\right)
$$

where

$$
\left\{\begin{array}{l}
V=\text { outward normal velocity of } \Gamma_{t} \\
\gamma=\text { ridge distance } \\
\kappa=\text { signed curvature. }
\end{array}\right.
$$

If $\kappa \leq 0$, the geometry changes, but a similar derivation leads also to (6.4), (6.5).

In summary, the free boundaries $\left\{\Gamma_{t}\right\}_{t \geq 0}$ marking the air/plastic interface evolve according to the nonlocal geometric evolution (6.4), at least provided that $\Gamma_{t}$, $u$, etc. are sufficiently smooth to justify the foregoing computations. A different derivation of formula (6.4) is to be found in Bergwall [B1, p. 29].

A general discussion of the evolution governed by (6.4) may be found in Feldman [F]. 


\section{Examples.}

We now utilize the rule $V=\gamma\left(1-\frac{\kappa \gamma}{2}\right)$ to work out some simple cases. The following selection of examples largely overlaps those in Bergwall [B1, B2], to whom we refer the reader for more details.

\section{A. Compressing a ring}

As a simple but instructive first explicit example, suppose $U_{0}$ is the annulus with inner radius $r_{0}$ and outer radius $R_{0}$. Then for small time $t>0, U_{t}$ is also annular, with inner radius $r$, outer radius $R$.

We have $\gamma=\frac{R-r}{2}$. Consequently the inward velocity of the hole is

$$
\begin{aligned}
V_{\text {in }}=\gamma\left(1-\frac{\kappa \gamma}{2}\right) & =\left(\frac{R-r}{2}\right)\left(1+\frac{1}{2 r}\left(\frac{R-r}{2}\right)\right) \\
& =\frac{R-r}{8}\left(3+\frac{R}{r}\right),
\end{aligned}
$$

which goes to infinity as $r \rightarrow 0$. The velocity of the outer edge is

$$
\begin{aligned}
V_{\text {out }}=\gamma\left(1-\frac{\kappa \gamma}{2}\right) & =\left(\frac{R-r}{2}\right)\left(1-\frac{1}{2 R}\left(\frac{R-r}{2}\right)\right) \\
& =\frac{R-r}{8}\left(3+\frac{r}{R}\right) .
\end{aligned}
$$

Assume the inner radius reaches 0 at time $t_{*}$. Then we have the ODE

$$
\begin{cases}\dot{r}=-\frac{R-r}{8}\left(3+\frac{R}{r}\right), & r(0)=r_{0} \\ \dot{R}=\frac{R-r}{8}\left(3+\frac{r}{R}\right), & R(0)=R_{0}\end{cases}
$$

for $0 \leq t<t_{*}$. In particular

$$
\lim _{t \rightarrow t_{*}^{-}} V_{\text {out }}=\frac{3}{8} R_{*}
$$

$R_{*}$ denoting the outer radius at $t_{*}$. Now for times $t \geq t_{*}, \gamma=R$ and therefore

$$
V_{\text {out }}=\gamma\left(1-\frac{\kappa \gamma}{2}\right)=\frac{R}{2}
$$

Hence

$$
\lim _{t \rightarrow t_{*}^{+}} V_{\text {out }}=\frac{R_{*}}{2}
$$

Comparing (7.1), (7.2) we see that our model predicts a instantaneous increase by $1 / 3$ in the outer velocity, at the time $t_{*}$ when the inner hole vanishes. At this moment the pressure $u$ is discontinuous, as $u(\cdot, t)=\operatorname{dist}_{+}\left(\cdot, \Gamma_{t}\right)$ at the center instantly changes from 0 to $R_{*}$. 
We are of course ignoring in this analysis the pressure of any air trapped within the hole.

\section{B. Motion of corners}

We next examine ramifications of the law $V=\gamma\left(1-\frac{\kappa \gamma}{2}\right)$ if the air/plastic interface has a corner. There are two possibilities, depending upon which way the corner points.

Outward pointing corner. In this case we interpret our rule $V=\gamma\left(1-\frac{\kappa \gamma}{2}\right)$ as predicting simply $V=0$. To justify this, observe that an outward oriented corner corresponds to $\kappa=+\infty$, and so we can understand the motion of $\Gamma_{t}$ at such a corner as the limiting behaviour for $\kappa \rightarrow+\infty$. But when $0<\kappa<\infty$, we have $0<\gamma \leq \frac{1}{\kappa}$. Thus the term $\kappa \gamma$ is bounded, whereas $\gamma \rightarrow 0$, as $\kappa \rightarrow+\infty$.

Inward pointing corner. Here we predict $V=+\infty$. Indeed, an inward pointing corner corresponds to $\gamma>0$ and $\kappa=-\infty$; so that the law $V=\gamma\left(1-\frac{\kappa \gamma}{2}\right)$ predicts a momentarily infinite outward velocity, which in turn immediately removes the corner.

Bergwall [B1] contains photographs of some experiments (for the compression of margarine) that support these conclusions.

Motion of a wedge. The simplest case illustrating the first effect is

$$
U_{0}=\left\{y>\lambda_{0}|x|\right\}
$$

with $\lambda_{0}>0$ (although this unbounded region is not strictly speaking covered by the theory before). We look for the subsequent plastic regions to have the form

$$
U_{t}=\{y>u(x, t)\}
$$

and derive a PDE for the height function $u$.

First of all, we may assume that, at least for small times $t \geq 0$, the ridge of $U_{t}$ is the positive $y$-axis. We locate the point $(0, z)$ on the $y$-axis for which the line segment connecting $(0, z)$ to $(x, u)$ is perpendicular to the tangent line to the graph of $u$ at $x$. That is,

$$
\left(\frac{u-z}{x}\right) u_{x}=-1
$$

Then

$$
\gamma=\left(x^{2}+(u-z)^{2}\right)^{\frac{1}{2}}=|x|\left(1+\frac{1}{u_{x}^{2}}\right)^{\frac{1}{2}}
$$

The curvature is

$$
\kappa=\frac{u_{x x}}{\left(1+u_{x}^{2}\right)^{\frac{3}{2}}}
$$

Hence

$$
\frac{u_{t}}{\left(1+u_{x}^{2}\right)^{\frac{1}{2}}}=-V=-\gamma\left(1-\frac{\kappa \gamma}{2}\right)
$$


and substituting above we discover

$$
u_{t}=\frac{x^{2} u_{x x}}{2 u_{x}^{2}}-\frac{|x|\left(1+u_{x}^{2}\right)}{u_{x}} .
$$

We look for a solution of (7.6) which is linear for $|x|$ for each time: $u=\lambda(t)|x|$. Plugging into (7.6) yields the ODE

$$
\lambda^{\prime}=-\frac{1+\lambda^{2}}{\lambda}
$$

Therefore

$$
u=\left(\left(1+\lambda_{0}^{2}\right) e^{-2 t}-1\right)^{\frac{1}{2}}|x|
$$

for $0 \leq t<t^{*}:=\frac{1}{2} \log \left(1+\lambda_{0}^{2}\right)$.

So the intial wedge of plastic moves by simply opening up, keeping straight sides, until it fills the entire upper half-plane at time $t^{*}$. In particular, the corner at the origin does not move at all until time $t^{*}$.

\section{Compressing a square}

A somewhat similar problem concerns the motion of the plastic/air interface when the intial configuration $U_{0}$ is the square with vertices at $( \pm 1, \pm 1)$. Inspired by the previous example, we suppose that the vertices do not move during some initial time period, and describe the upper, righthand edge of $U_{t}$ by the height function $u=u(x, t)$ for $0 \leq x \leq 1$.

We assume as well that at least for some time, the ridge of $U_{t}$ consists of the two diagonals $\{(z, \pm z) \mid-1 \leq z \leq 1\}$.

Given then $(x, u) \in \Gamma_{t}$, we look for the point $(z, z)$ on the main diagonal for which the line segment connecting to $(x, u)$ is perpendicular to the tangent line to the graph of $u$. This means

$$
\left(\frac{u-z}{x-z}\right) u_{x}=-1
$$

Then

$$
\gamma=\left((x-z)^{2}+(u-z)^{2}\right)^{\frac{1}{2}}=(u-z)\left(1+u_{x}^{2}\right)^{\frac{1}{2}}=\frac{u-x}{1+u_{x}}\left(1+u_{x}^{2}\right)^{\frac{1}{2}}
$$

Since

$$
\frac{u_{t}}{\left(1+u_{x}^{2}\right)^{\frac{1}{2}}}=V=\gamma\left(1-\frac{\kappa \gamma}{2}\right)
$$

and the curvature is given by (7.5), we derive after some substitutions the PDE

$$
u_{t}=\frac{1}{2}\left(\frac{u-x}{1+u_{x}}\right)^{2} u_{x x}+\frac{(u-x)\left(1+u_{x}^{2}\right)}{1+u_{x}}
$$

holding so long as $u_{x}>-1$. The corresponding boundary conditions are

$$
u_{x}(0, t)=0, u(1, t)=1 \text {. }
$$


We conjecture that the height function $u$ obeys (7.7), (7.8), until a time $t^{*}>0$ at which $u_{x}\left(1, t^{*}\right)=-1$. At this moment the corner at the vertex $(1,1)$ should have straightened out and the air/plastic edge there should begin to move. The reasoning above is not valid after time $t^{*}$.

\section{Compression near a flat obstacle}

As an interesting variant of the foregoing examples, assume next that our compressed polymer is constrained by an obstacle to lie in the upper halfplane. We assume also that

$$
U_{t}=\{0 \leq y<u(x, t)\}
$$

so that $U_{t}$ lies beneath the graph of a nonnegative height function $u$. Supposing as well that $u$ is smooth, we suggest that the lower edge $\{y=0\}$ should act like the ridge set in the dynamical law $V=\gamma\left(1-\frac{\kappa \gamma}{2}\right)$.

Again the goal is finding a differential equation for $u$, and for this we follow Bergwall $[\mathrm{B} 1, \S 6.1]$. As in our earlier examples, we seek $(z, 0)$ so that the line segment from this point to $(x, u)$ is perpendicular to the tangent line. Then

$$
\left(\frac{u}{x-z}\right) u_{x}=-1
$$

Continuing as before, we eventually arrive at the PDE

$$
u_{t}=\frac{1}{2} u^{2} u_{x x}+u u_{x}^{2}+u=\left(\frac{u^{3}}{6}\right)_{x x}+u
$$

This is a form of the porous medium equation, with an extra source term $u$ on the right. Bergwall [B1] contains this derivation, in the case of a free compression that is symmetric across the $x$-axis. The reader should refer to [B1] for more about this analogy with the porous medium equation.

\section{More geometric properties of the free boundary.}

This concluding section provides some formal computations for general flows of smooth, open subsets $\left\{U_{t}\right\}_{t \geq 0}$ in the plane, whose boundaries $\left\{\Gamma_{t}\right\}_{t \geq 0}$ move according to our nonlocal law $V=\gamma\left(1-\frac{\kappa \gamma}{2}\right)$. It is an interesting open question to extend the following calculations to the generalized flow built in $\S 4,5$.

\section{A. A formula for area}

To start, we first observe that if $U$ is a smooth, bounded, open subset of the plane, we have the geometric identity

$$
|U|=\int_{\partial U} \gamma\left(1-\frac{\kappa \gamma}{2}\right) d \mathcal{H}^{1}
$$


See Bergwall [B1, p.28] for a derivation of this formula.

\section{B. Rate of change of area}

If now $U_{t}$ represents a smoothly evolving region whose boundary $\Gamma_{t}$ at each moment moves according to our nonlocal law $V=\gamma\left(1-\frac{\kappa \gamma}{2}\right)$, we can compute

$$
\frac{d}{d t}\left|U_{t}\right|=\int_{\partial U_{t}} V d \mathcal{H}^{1}=\int_{\partial U_{t}} \gamma\left(1-\frac{\kappa \gamma}{2}\right) d \mathcal{H}^{1}=\left|U_{t}\right|,
$$

and so

$$
\left|U_{t}\right|=e^{t}\left|U_{0}\right| \quad(t \geq 0)
$$

This deduction is consistent with the rigorous theory from $\S 4,5$.

\section{Rate of change of boundary length}

Remember that $\mathcal{H}^{1}$ denotes one-dimensional Hausdorff measure, so that $\mathcal{H}^{1}\left(\Gamma_{t}\right)$ is the length of the boundary $\Gamma_{t}$. Then

$$
\begin{aligned}
\frac{d}{d t}\left(\mathcal{H}^{1}\left(\Gamma_{t}\right)\right) & =\int_{\Gamma_{t}} V \kappa d \mathcal{H}^{1} \\
& =\int_{\Gamma_{t}} \kappa \gamma\left(1-\frac{\kappa \gamma}{2}\right) d \mathcal{H}^{1} \\
& =\frac{1}{2} \mathcal{H}^{1}\left(\Gamma_{t}\right)-\frac{1}{2} \int_{\Gamma_{t}}|\gamma \kappa-1|^{2} d \mathcal{H}^{1}
\end{aligned}
$$

Consequently,

$$
\mathcal{H}^{1}\left(\Gamma_{t}\right) \leq e^{\frac{t}{2}} \mathcal{H}^{1}\left(\Gamma_{0}\right) \quad(t \geq 0)
$$

\section{Evolving isoperimetric ratios}

Next we calculate that

$$
\begin{aligned}
\frac{d}{d t}\left(\frac{\mathcal{H}^{1}\left(\Gamma_{t}\right)^{2}}{\left|U_{t}\right|}\right) & =2 \frac{\mathcal{H}^{1}\left(\Gamma_{t}\right)}{\left|U_{t}\right|} \frac{d}{d t}\left(\mathcal{H}^{1}\left(\Gamma_{t}\right)\right)-\frac{\mathcal{H}^{1}\left(\Gamma_{t}\right)^{2}}{\left|U_{t}\right|^{2}} \frac{d}{d t}\left|U_{t}\right| \\
& =2 \frac{\mathcal{H}^{1}\left(\Gamma_{t}\right)}{\left|U_{t}\right|}\left(\frac{1}{2} \mathcal{H}^{1}\left(\Gamma_{t}\right)-\frac{1}{2} \int_{\Gamma_{t}}|\gamma \kappa-1|^{2} d \mathcal{H}^{1}\right)-\frac{\mathcal{H}^{1}\left(\Gamma_{t}\right)^{2}}{\left|U_{t}\right|} \\
& =-\frac{\mathcal{H}^{1}\left(\Gamma_{t}\right)^{2}}{\left|U_{t}\right|} \int_{\Gamma_{t}}|\gamma \kappa-1|^{2} d \mathcal{H}^{1}
\end{aligned}
$$

where we employed $(8.2),(8.4)$ and the slash through the integral means average:

$$
f_{\Gamma_{t}}|\gamma \kappa-1|^{2} d \mathcal{H}^{1}:=\frac{1}{\mathcal{H}^{1}\left(\Gamma_{t}\right)} \int_{\Gamma_{t}}|\gamma \kappa-1|^{2} d \mathcal{H}^{1} .
$$


In view of $(8.6)$,

$$
\frac{\mathcal{H}^{1}\left(\Gamma_{t}\right)^{2}}{\left|U_{t}\right|}=\frac{\mathcal{H}^{1}\left(\Gamma_{0}\right)^{2}}{\left|U_{0}\right|} \exp \left(-\int_{0}^{t} f_{\Gamma_{s}}|\gamma \kappa-1|^{2} d \mathcal{H}^{1} d s\right) .
$$

But the isoperimetric inequality states

$$
\frac{\mathcal{H}^{1}\left(\Gamma_{t}\right)^{2}}{\left|U_{t}\right|} \geq 4 \pi
$$

whence (8.7) gives the estimate

$$
\exp \left(\int_{0}^{t} f_{\Gamma_{s}}|\gamma \kappa-1|^{2} d \mathcal{H}^{1} d s\right) \leq \frac{\mathcal{H}^{1}\left(\Gamma_{0}\right)^{2}}{4 \pi\left|U_{0}\right|} \quad(t \geq 0)
$$

We interpret this inequality to imply that the (rescaled) boundaries $\left\{\Gamma_{t}\right\}_{t \geq 0}$ converge to circles as $t \rightarrow \infty$. 


\section{References}

[Am] L. Ambrosio, Lecture notes on optimal transport problems, Preprint: Scuola Normale Superiore (2000).

[A] G. Aronsson, Asymptotic solution of a compression molding problem, Preprint LiTH-MAT-R95-1, Department of Mathematics, Linköping University (1995).

[A-E-W] G. Aronsson, L. C. Evans and Y. Wu, Fast/slow diffusion and growing sandpiles, Journal of Differential Equations 131 (1996), 304-335.

[A-J] G. Aronsson and U. Janfalk, On Hele-Shaw flow of power-law fluids, European J. Appl. Math 3 (1992), 343-366.

[B1] A. Bergwall, A geometric evolution problem arising in an asymptotic approach to compression molding, Linköping Studies in Science and Technology, Dissertation \#693, Linköping University (1998).

[B2] A. Bergwall, A geometric evolution problem arising in an asymptotic approach to compression molding, to appear in Quarterly of Applied Mathematics.

[C-F-M] L. Caffarelli, M. Feldman and R. McCann, Constructing optimal maps for Monge's transport problem as a limit of strictly convex costs, to appear.

[E-J] C. M. Elliott and V. Janovsky, A variational inequality approach to Hele-Shaw flow with a moving boundary, Proc Royal Soc Edinburgh 88 (1981), 91-107.

[E-O] C. M. Elliott and J.R. Ockendon, Weak and variational methods for moving boundary problems, Pitman Research Notes in Mathematics \# 59, 1982.

[E] L. C. Evans, Partial differential equations and Monge-Kantorovich mass transfer, Current Developments in Mathematics, 1997.

[E-G1] L. C. Evans and W. Gangbo, Differential equations methods in the Monge-Kantorovich mass transfer problem, Memoirs American Math. Society \#654 137 (1999).

[E-G2] L. C. Evans and R. F. Gariepy, Measure Theory and Fine Properties of Functions, Second Edition, CRC Press, 1992.

[F] M. Feldman, Variational evolution problems and nonlocal geometric motion, Arch. Rat. Mech. Analysis 146 (1999), 221-274.

[F-L-T] F. Folgar, C.-C. Lee and C. L. Tucker, Simulation of compression molding for fiber-reinforced thermosetting polymers, Trans. ASME J. of Eng. for Industry 106 (1984), 114-125.

$[\mathrm{K}] \quad$ J. R. King, Development of singularities in some moving boundary problems, European J of Applied Math 6 (1995), 491-507.

[G-T] D. Gilbarg and N. Trudinger, Elliptic Partial Differential Equations of Second Order, 2nd ed, Springer, 1983.

[O-T] T. Osswald and C. Tucker, A boundary element simulation of compression mold filling, Polymer Eng. and Sci. 28 (1988).

[J1] U. Janfalk, Behaviour in the limit, as $p \rightarrow \infty$, of minimizers of functionals involving p-Dirichlet integrals, SIAM Journal Math Analysis 27 (1996), 341-360.

[J2] U. Janfalk, On a minimization problem for vector fields in $L^{1}$, Bulletin London Math Society 28 (1996), 165-176. 